Document donwnloaded from:

[http://redivia.gva.es/handle/20.500.11939/6684]

This paper must be cited as:

[Asins, M.J., Raga, M.V., Roca, D. et al. (2020). QTL and candidate gene analyses of rootstockmediated mandarin fruit yield and quality traits under contrasting iron availabilities. Tree Genetics \& Genomes 16, 79 ]

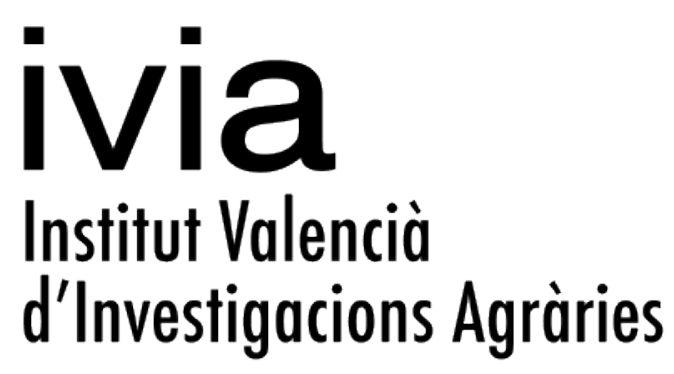

The final publication is available at

[http://dx.doi.org/10.1007/s11295-020-01472-w]

Copyright [Springer] 


\title{
QTL and candidate gene analyses of rootstock-mediated mandarin fruit yield and quality traits under contrasting iron availabilities
}

Maria J. Asins*, M. Verónica Raga, Dolors Roca, Emilio A. Carbonell

Instituto Valenciano de Investigaciones Agrarias, Carretera de Moncada a Náquera Km 4.5, Apartado Oficial, 46113 Moncada, Valencia, Spain.

* Corresponding author; email: mjasins@ivia.es Telephone: 34963424067

\section{https://orcid.org/0000-0002-4330-160X}

\begin{abstract}
The most sustainable approach to overcome iron deficiency in fruit crops is breeding for rootstocks with a higher capability to acquire iron $(\mathrm{Fe})$ from the soil. The objective of this study was quantitative trait loci (QTL) and candidate gene analyses of rootstock-mediated low-Fe tolerance in terms of fruit yield and quality traits, including Fe fruit content, in a satsuma mandarin-grafted rootstock population derived from a cross between Citrus reshni (Cleopatra mandarin) and Poncirus trifoliata, under sufficient and low-Fe fertilization (15.3 vs $5.2 \mu \mathrm{M} \mathrm{Fe}$, respectively).

Iron reduction to one third significantly decreased satsuma leaf chlorophyll concentration, fruit iron concentration, and the fruit/leaf iron proportion. Thirty-four QTLs were detected for 46 heritable traits. Eighteen of them were also found significant when testing each parental genome separately. Seven QTLs contributed to the fruit concentrations of $\mathrm{Cu}, \mathrm{Fe}, \mathrm{K}, \mathrm{Na}$, and S. QTLs involved in rootstock mediated tolerance to Fe deficiency and fruit quality traits distributed into five genomic regions whose gene contents (assuming collinearity with the C. clementina genome) were investigated for overrepresented molecular functions and biological processes, and putative functional candidates. Among them, a metal-NA-transporter YSL3 (Ciclev 10019170m), four phytochelatin synthases, an iron-chelate-transporter ATPase, and four basic/helix-loop helix genes coding for likely relevant transcription factors in $\mathrm{Fe}$ homeostasis under $\mathrm{Fe}$ deficiency were found: bHLH3 (Ciclev10019816m), bHLH137.1 (Ciclev10031873m), bHLH123 (Ciclev10008228m) and ILR3 (Ciclev10009354m). Genes within three QTL regions supported a genetic connection between rootstock-mediated tolerance to $\mathrm{Fe}$ deficiency and biotic stresses in citrus.
\end{abstract}

Keywords: Rootstock breeding, Iron deficiency, Citrus reshni, Poncirus trifoliata, Citrus unshiu, Disease resistance. 


\section{Introduction}

Iron $(\mathrm{Fe})$ is abundant in the soil but it is usually present in an oxidized form, difficult to be acquired by plants. Besides, in alkaline soils which affects around $30 \%$ of the earth, Fe solubility is low leading to iron deficiency chlorosis (Mengel 1994). Iron deficiency causes decreases in fruit yield and quality (Almaliotis et al. 1995). Besides, soil Fe deficiency might decrease fruit Fe content, affecting human nutrition, health and well-being (Rashid and Ryan 2004). Iron sulfate and synthetic chelates are commonly used as iron fertilizers to overcome Fe deficiency (Jessop et al. 1990; Abadia et al. 2004) but they are not fully efficient due to their rapid transformation into an unavailable form in the calcareous soil (Fernandez et al. 2004), and increase orchard management costs. Therefore, the best, cost-effective and sustainable approach is breeding for rootstocks with a higher capability to acquire Fe from the soil. Many citrus rootstocks are limited by their inability to sufficiently extract iron and other micronutrients from calcareous soils (Korcak 1987; Manthey et al. 1994) what has motivated numerous studies on citrus germplasm evaluation for tolerance to low-Fe stress (Castle 1987; Castle et al. 2009; Pestana et al. 2011, 2005). In the greatest effort, Castle et al. (2009) provided the following order of rootstocks in decreasing degree of tolerance: Volkamer lemon/Rangpur/sour orange selections/Citrus macrophylla $>$ mandarins and mandarin hybrids $>$ citranges $>$ citrumelos $>$ trifoliate orange.

From the agronomic point of view, tolerance to low-Fe stress should be considered in terms of fruit yield and quality, however this type of evaluation is extremely lengthy and costly due to the long juvenility of trees, and the need to identify nucellar seedlings to be grafted with a commercial variety (Raga et al. 2012, 2016; Huang et al. 2018). These varieties mostly correspond to sweet oranges (Citrus sinensis (L.) Osb), mandarins (mainly Citrus clementina Hort. ex Tan.and Citrus unshiu (Mak.) Marc.), grapefruits (Citrus paradise Macf.), pummelos (Citrus grandis (L.) Osb.), and lemons (Citrus limon L. Burm. f.). Cultivars of all these species are always vegetatively propagated by bud grafting onto a seedling rootstock in order to obtain a more uniform and earlier yielding tree with tolerance to pathogens and well adapted to the local edaphoclimatic conditions. Mandarin fruits are excellent sources of vitamin C, mineral elements and provide two important antioxidant phytochemicals: beta-carotene and beta-cryptoxanthin (Lado et al. 2016). Citrus ( $2 n=18$ chromosomes) is mostly cultivated in arid and semi-arid areas, some of them, such as the Mediterranean area, are extensively deficient in iron (Jaegger et al. 2000). The analysis of quantitative trait loci 
(QTL) governing rootstock-mediated fruit yield and quality traits under contrasting iron levels would be useful to implement marker-assisted selection schemes in rootstock breeding programs and search for functional candidate genes underlying such QTL.

Trying to understand the molecular mechanisms behind citrus adaptation and tolerance to low-Fe stress, several authors have studied root transcriptional and proteomic differences between iron chlorosis tolerant and susceptible citrus rootstocks under contrasting iron fertilization conditions (Fu et al. 2017, Licciardello et al. 2013, Muccilli et al. 2013), the metabolic and molecular changes that take place in citrus under iron deficiency (Martinez-Cuenca et al. 2013, Fu et al. 2017), and recently, Zhang et al. (2020) have provided a reduced list of 14-21 members of the basic/helix-loophelix (bHLH) transcription factor family as putative key regulators of the iron deficiency response in $C$. grandis.

Here we present a pioneering genetic study of rootstock effects on a grafted mandarin using a Citrus $\times$ Poncirus population. The objectives were the QTL and candidate gene analyses of rootstock-mediated low-Fe tolerance in terms of fruit yield and quality traits, particularly fruit iron content, using a progeny derived from two wellknown citrus rootstocks, Cleopatra mandarin (Citrus reshni Hort. ex. Tan.) and trifoliate orange (Poncirus trifoliata (L.) Raf.) which were previously reported to differ in tolerance to low-Fe stress (Castle et al. 2009). The segregating population was originated by nucellar-seedling propagation from 62 apomictic hybrids of a reference population (151 hybrids) that had been previously genotyped (Raga et al. 2012). In the present study we have anchored an integrated $C$. reshnni-P. trifoliata genetic linkage map to the physical map of $C$. clementina, the most closely related species to $C$. reshni (Herrero et al. 1996) whose sequence is available, to approach an intensive candidate gene analysis within relevant QTL regions taking advantage of citrus databases (phytozome.jgi.doe.gov, citrus.hzau.edu.ch, ncbi.nlm.nih.gov) and bioinformatic tools.

\section{Materials and Methods}

\section{Plant material}

A mapping population that consists of 151 hybrids $(\mathrm{R} \times \mathrm{Pr})$ previously genotyped (Raga et al. 2012) was used to identify apomictic hybrids (Raga et a. 2016) that were propagated through nucellar seedlings and grafted for the present experiment. The process and steps towards the obtention of both mapping and phenotyped populations are graphically described in Figure 1 . The mapping population was obtained at IVIA (Valencia, Spain) by controlled crosses between Citrus reshni Hort. ex. Tan. (Cleopatra 
mandarin) as female (salt and iron chlorosis tolerant and apomictic) parent, and two apomictic and disease resistant varieties of Poncirus trifoliata (L.) Raf. (trifoliate orange): Flying Dragon (83 hybrids) and Rich (68 hybrids) as pollinators. Seedlings of the next generation were analyzed by molecular markers to discard the zygotic ones (Ruiz et al. 2000). Finally, nucellar seedlings obtained from the $62 \mathrm{R} \times \mathrm{Pr}$ hybrids that showed apomictic reproduction and parents (Cleopatra and Flying Dragon) were grafted with Clausellina mandarin (Citrus unshiu (Mak.) Marc.) and maintained for more than 5 years till full production before the experiment.

\section{Growth conditions}

Two-Three out of six repetitions (nucellar grafted plants) of each $\mathrm{R} \times \mathrm{Pr}$ hybrid were randomly selected to establish two treatments: control-sufficient $(15.3 \mu \mathrm{M} \mathrm{Fe})$ and low-Fe treatment $(5.2 \mu \mathrm{M} \mathrm{Fe})$, during 9 months (from February till November) in a greenhouse. Plants were growing into pots $(17 \mathrm{~L})$ using cocofiber as a substrate. The greenhouse had automatic roof ventilation and heating system (maintaining inside air temperature above $8^{\circ} \mathrm{C}$ ). A high frequency fertirrigation system together with $4 \mathrm{~L} / \mathrm{h}$ drippers were used and handled to ensure homogeneity of low $[\mathrm{Fe}]$ at the roots of all plants in cultivation at the same time. The nutrient solution (pH: 6.4) contained the following concentration of macronutrients (in $\mathrm{mM}$ ): $\mathrm{NO}_{3}{ }^{-} 8.1 ; \mathrm{H}_{2} \mathrm{PO}_{4}{ }^{-} 4 ; \mathrm{SO}_{4}{ }^{2-} 1 ; \mathrm{NH}_{4}{ }^{+}$ 0.9; $\mathrm{K}^{+} 4.2 ; \mathrm{Ca}^{2+} 3.5 ; \mathrm{Mg}^{2+} 1$; plus, the following concentration of micronutrients (in $\mu \mathrm{M}): \mathrm{Mn}^{2+} 8 ; \mathrm{Zn}^{2+} 2.3$; $\mathrm{B} 20, \mathrm{Cu}^{2+} 7 ; \mathrm{Mo}^{4+} 0.5$ and $\mathrm{Fe}^{2+} 15.3$ or 5.2 depending on the treatment (control or low-Fe, respectively). The water for the nutrient solution was previously treated with reverse osmosis.

\section{Trait evaluation}

Several vegetative, physiological and agronomic (related to fruit yield and quality) traits were evaluated on the grafted satsuma variety (see Table 1 for the abbreviation list) under both control and low-Fe conditions, denoted by $\mathrm{C}_{-}$and $\mathrm{Fe}_{-}$ prefixes, respectively. Chlorophyll leaf concentration of fully expanded young leaves from each plant (S3) was estimated with the chlorophyll meter SPAD-502 Plus (Konica Minolta, INC., Japan) after 3 months of treatment. Three fully developed leaves per plant were sampled from vegetative spring shoots after 8 months of treatment to measure the following leaf characteristics: leaf fresh weight (LFW, g); leaf dry weight (LDW, g) measured in samples dried at $80^{\circ} \mathrm{C}$ for 3 days, leaf water content (LWC, g) as the difference between fresh and dried weights, leaf dry matter (LDM, \%) calculated 
as the percentage of LDW to LFW, and Leaf area [LA (square centimeter)] measured with a leaf area quantifier (LI-3100C area meter; LI-Cor, Lincoln, NE).

A minimum of 5 randomly sampled fruit per tree also were evaluated for the following internal fruit-quality traits: fruit weight ( $\mathrm{FW}$, in $\mathrm{g})$; fruit diameter $(\mathrm{FD}$, in $\mathrm{mm})$; rind thickness (RT, in $\mathrm{mm})$; juice volume per fruit ( $\mathrm{JV}$, in $\mathrm{mL}$ ) without pulp, juice content (JC, percentage from JV and FW), soluble-solids content [SSC, as ${ }^{\circ}$ Brix, using a digital refractometer (Pallete PR-101; Atago, Tokyo, Japan)], juice acidity measured as volume of $\mathrm{NaOH} 0.1 \mathrm{M}$ to neutralize acidity per fruit $(\mathrm{NaOH}$, in $\mathrm{mL}$, using phenolphthalein indicator), and maturation index (SSC/A, as the ratio between SSC and the percentage of citric acid calculated from $\mathrm{NaOH}$ ).

Dry tissue samples of the fruit raw edible part (F), and leaf (Lf), were prepared for mineral analysis by digestion in a $\mathrm{HNO}_{3}: \mathrm{HClO}_{4}(2: 1$, v/v) solution. Inorganic solutes were determined in ppm $(\mathrm{mg} / \mathrm{Kg})$ by inductively coupled plasma spectrometry (Varian ICP 720-E, Scientific Instrumentation Service, Estación Experimental del Zaidín, CSIC, Granada, Spain). These traits were named by the element symbol followed by F or Lf, denoting the tissue. Inorganic solutes were also determined in the leaf at the beginning of the low-Fe treatment. Thus, the change (accumulation or loss) of each element concentration, denoted by the prefix d, was estimated as the difference between its final and initial leaf concentrations. The relative Fe_F to Fe_Lf was also considered (Fe_F/L) as percentage, and also the relative Al_F to Al_Lf (Al_F/L).

Fruit yield was evaluated in terms of number of normal, ripe fruits (FNm), their individual weight, (FWm, g) and total fruit weight (TFWm, Kg). Total dry fruit weight (TDFWp, g) was estimated from the mean dry weight of fruit pulp and FNm, Finally, total harvested Fe (mg of Fe in total fruit yield) was deduced from TDFWp and Fe_F. This trait, coded as FeUEp, could be considered as a comparative, agronomic indicator of the rootstock iron uptake and translocation capacity, under both Fe levels (Asins et al. 2020). Similar estimations for P, S and Mg fruit contents (PUEp, SUEp and MgUEp) were also obtained.

\section{Statistical analysis}

Pearson correlation coefficients and principal component analysis based on the correlation matrix for the adjusted means were used to study the relations between the different traits.

The experiment was designed as a split-plot with four blocks using iron treatments as the main plot and rootstocks as the subplots. The statistical analysis of the 
experiment followed this experimental design, i.e. blocks were random, and to study the $\mathrm{G} \times \mathrm{E}$ interaction the effects of genotype and treatment were classed as fixed. Considering $\mathrm{R} \times \mathrm{Pr}$ hybrid genotypes as a random effects factor, broad-sense heritability $\left(\mathrm{H}^{2}\right)$ was estimated for all traits for nucellar rootstocks (repetitions) derived from apomictic $\mathrm{R} \times \operatorname{Pr}$ hybrids under control or low-Fe conditions, based on the genotypic $\left(\mathrm{V}_{\mathrm{G}}\right)$ and environmental $\left(\mathrm{V}_{\mathrm{E}}\right)$ variance estimators calculated by minimum variance quadratic unbiased estimator (MIVQUE), as previously reported (Villalta et al. 2007).

Molecular markers, QTL and candidate gene analyses

QTL analyses were carried out using the genotypic and map data from Raga et al. (2012) based on SSR, IRAP and SCAR markers, and the adjusted means of traits. Interval Mapping (IM) procedure in MapQTL ® 6 (Van Ooijen 2009), and Multiple QTL Mapping (MQM) when more than one QTL was detected in the same linkage group were used to identify QTLs. QTL analyses were carried out in two different ways. First, we analyzed the data as a cross-pollinated (CP) population type in order to consider intralocus interaction and second, we analyzed data for each parental meiosis separately; i.e. a "two-way pseudo-testcross" analysis (Grattapaglia and Sederoff 1994). This second approach provides the computation advantages of the two-genotypes QTL model but the disadvantage of losing power (and reality) because intralocus interaction is ignored (Van Ooijen 2009). JoinMap 4.1 (Van Ooijen 2012) was used to translate and split the marker data to separate the two meiosis. Some linkage groups or linkage group parts (R9a, R6, R4a, Pr1, Pr4a, and Pr9b) were parent-specific; so they were ignored when using the CP data for QTL analysis. Cleopatra map contained 86 markers, distributed along 10 linkage groups, covering $1127.127 \mathrm{cM}$ of the $C$. reshni genome. Similarly, Poncirus map contained 73 markers, distributed along 11 linkage groups, covering $1416.759 \mathrm{cM}$ of the Poncirus trifoliata genome. The CP map contained 93 markers, distributed along 9 linkage groups, covering $1406.761 \mathrm{cM}$ of the integrated genome.

For IM and MQM, a 5\% experimentwise significance level was assessed by permutation tests. These LOD critical values ranged from 1.1 to 2.0 depending on the trait and linkage group in the "two-way pseudo-testcross" analysis (population type $\mathrm{DH})$. On the other hand, the LOD critical values ranged from 2.2 to 3.3 depending on the trait and linkage group in in the CP analysis. Only significant QTLs with LOD $\geq 2.38$ for heritable traits $\left(\mathrm{H}^{2}>0\right)$ are reported here. 
A two-way ANOVA was used to study the interaction (epistasis) between markers corresponding to QTLs controlling some traits (Fe_Cu_F, Fe_S3 and Fe_FeUEp).

Some genomic regions were particularly rich in QTLs or had QTLs for relevant

205

206

207

208

209

210

211

212 traits. For these regions, markers from the $\mathrm{CP}$ map were anchored to the physical map of C. clementina using primer and/or EST sequences and the BLASTN tool (https://phytozome.jgi.doe.gov/pz/portal.html\#!search?show=BLAST). Genes covering one LOD decay at both sides of the QTL peaks were downloaded from C. clementina genome at https://phytozome.jgi.doe.gov. C. clementina was chosen instead of $C$. sinensis because Cleopatra mandarin is genetically closer to $C$. clementina than to $C$. sinensis (Herrero et al. 1996, Wu et al. 2018). The annotation of some genes downloaded from phytozome.jgi.doe.gov was tested by blasting their peptide sequence at ncbi.nlm.nih.gov. Gene ontology (GO) enrichment analysis of genes within one LOD intervals of QTLs were carried out using the Singular Enrichment Analysis tool (Tian et al. 2017) at the AgriGo platform (http://systemsbiology.cau.edu.cn/agriGOv2/).

\section{Results}

The mean (and standard error) of the phenotypic values of controls (Cleopatra mandarin and Flying Dragon parents) for the analyzed traits under Fe-sufficient and low-Fe treatments are presented in Table 1. Significant differences between the parents were detected for a few traits under one Fe level, particularly for S3 (SPAD) under lowFe treatment (Online Resource 1) where Clausellina leaves grafted on Flying Dragon showed higher chlorophyll content than those on Cleopatra $(73.90 \pm 1.70$ versus $49.25 \pm 18.85$, respectively, in Table 1). The pulp of Clausellina mandarin fruits was found particularly rich in K, followed by $\mathrm{P}, \mathrm{Ca}$ and $\mathrm{S}$. Silicon was also present and its amount was similar to that of iron for the parental rootstocks under low-Fe stress (20.14 \pm 5.04 and 19.32 \pm 0.64 ppm, for Cleopatra and Flying Dragon, respectively). Although with the least concentration, aluminium was present in the fruit, particularly under low-Fe condition for Cleopatra (6.96 $\pm 4.9 \mathrm{ppm})$.

The range of variation in the grafted rootstock-segregating population and the estimated heritabilities under sufficient $\left(\mathrm{H}^{2}{ }_{-} \mathrm{C}\right)$ and low-Fe fertilization $\left(\mathrm{H}^{2}{ }_{-} \mathrm{Fe}\right)$ are also included in Table 1. Heritability estimates of some traits $\left(\mathrm{Cu}_{-} \mathrm{F}, \mathrm{K} \_\mathrm{F}, \mathrm{P} \_\mathrm{F}, \mathrm{Fe} \_\mathrm{Lf}\right.$, $\mathrm{Na} \_$Lf, fruit juice tritable acidity $(\mathrm{NaOH}), \mathrm{TDFWp}$ and related traits), notably increased under low-Fe. 
A summary of results from the mixed model analysis of the segregating population is provided in Online Resource 2. Leaf [Ca] was the only trait where significant $\mathrm{G} \times \mathrm{E}$ interaction was detected in the experiment. Iron reduction to one third significantly decreased leaf chlorophyll concentration (S3), fruit iron concentration $\left(\mathrm{Fe} \_\mathrm{F}\right)$ and the fruit/leaf iron proportion (Fe_F/Lf) (Online Resource 2, Figure 2).

The relationships among traits evaluated under control and low-Fe are graphically represented in Figures 3 and 4, respectively. As expected, most fruit yield traits form a group of strong positive correlations, and negatively related to the group formed by $\mathrm{FW}$ and JV. Fruit concentrations of S, K and $\mathrm{P}$ are also strongly related forming a conserved group between treatments. Fruit yield traits FNm, TFWm, TDFWp, and PUEp were significantly correlated under control and low-Fe, as well as leaf concentrations of $\mathrm{Al}, \mathrm{K}, \mathrm{Mg}, \mathrm{P}, \mathrm{S}$ and $\mathrm{Si}$ (Online Resource 3). Additional information on significantly $(\mathrm{p}<0.02)$ correlated traits is given in Online Resource 4. The only elements whose concentrations in leaf and fruit were correlated under both treatments were $\mathrm{Na}$ and $\mathrm{Mn}$ ( $\mathrm{P}$, only under control condition). In the case of $\mathrm{Mn}$, this correlation increased from 0.38 (control) up to 0.63 (low-Fe). Noteworthy, TDFWp and Fe_F were negatively related only under control conditions.

Main features of the 34 significant QTLs detected for 46 heritable traits are shown in Table 2. Eighteen of those QTLs were also found significant when testing each parental genome separately: 11 at the Cleopatra mandarin map, and 7 at the Poncirus trifoliata map. Except for fruit yield QTLs FNm and TFWm on linkage group $4 \mathrm{~b}$, that were detected under both treatments, the rest are condition-specific. Several rootstock QTLs were found to contribute to the fruit concentrations of $\mathrm{Fe}$ and $\mathrm{K}$ under control, and of $\mathrm{Cu}, \mathrm{Na}$ and $\mathrm{S}$ under low-Fe fertilization. Epistatic interactions were detected for two traits: $\mathrm{Cu}$ fruit concentration under low $\mathrm{Fe}$ (between $\mathrm{Fe} \_\mathrm{Cu}$ _F QTLs markers CR17,300 and C8iC1rt,650; Figure 5A), and SPAD values under low Fe (between Fe_S3 QTLs markers CT19.165 and C1,1600; Figure 5B). In all of them, one of the genotypic combinations should be avoided through selection in rootstock breeding programs.

Five genomic regions, four of them showing clustering of QTLs were further analyzed for candidate genes (Table 2 and Figure 6). For this purpose, all markers at these QTL regions were first anchored at the C. clementina physical map. A QTL for fruit concentration of $\mathrm{Cu}$ was at region I on LG 7 (Scaffold 3); Fruit yield QTLs FNm, and TFWm formed a cluster (IV) on LG 4b (Scaffold 7); and Fe, Mg, S, and P total fruit 
contents under low-Fe formed a cluster (II) on LG 7 (Scaffold 3) and another (III) on LG 12 (Scaffold 4). Cluster V corresponded to QTLs for S3, and leaf change of K and $\mathrm{Cu}$ concentrations on LG $3 b$ (Scaffold 1). Physical distances in Mbp around the QTL peaks (around 1 LOD) were estimated to download the genes (mRNA) included. Enrichment analysis of these genes resulted in significant Biological Processes for clusters II (signaling), IV (DNA damage checkpoint), and V (phosphorylation, cell recognition); and significant Molecular Functions for regions I (nutrient reservoir activity, GO:0045735, FDR=0.026), II (peptidase inhibitor activity), IV (ADP binding, ATPase activity), and V (protein Ser/Thr kinase activity) (Online Resource 5).

A summary list of candidate genes is shown in Online Resource 6. Among them, a metal-NA-transporter YSL3 (Ciclev 10019170m) was found within cluster II, four phytochelatin synthases were within region I, and an iron-chelate-transporter ATPase in cluster IV. Four basic/helix-loop helix genes coding for likely relevant transcription factors in Fe homeostasis under Fe deficiency (Zhang et al. 2020) were found in clusters I (bHLH3, Ciclev10019816m), III (bHLH137.1, Ciclev10031873m), and V (bHLH123, Ciclev10008228m; and ILR3, Ciclev10009354m). Some genes related to phytohormone metabolism/signaling were found within cluster I (ethylene, auxin), II (ethylene), III (ethylene, polyamines, salicylic acid, auxin), IV (ethylene, gibberellin, brassinosteroid) progenies

In spite of the importance of the rootstock in citriculture, up to our knowledge, no genetic analysis of rootstock effects on fruit traits has been reported yet. Two wellknown reasons are the long juvenility of Citrus $\times$ Poncirus hybrids and the segregation for apomictic reproduction in these populations (Raga et al. 2012). Thus, although the present study is based on a genetic linkage map previously obtained from the whole $C$. reshni $\times$ Poncirus trifoliata progeny (151 trees; Raga et al. 2012), only 62 showed the needed apomictic reproduction to be used as rootstock (Fig. 1). Therefore, a limitation of this study is the number phenotyped genotypes because not all of them could be replicated through nucellar seedlings and grafted for phenotypic evaluations. Under this circumstance, QTL resolution is not limited by marker density but by the size of the phenotyped subpopulation. Recent QTL studies in Citrus $\times$ Poncirus segregating 
populations (Lima et al. 2018; Huang et al. 2018) have used saturated linkage maps, mostly based on SNPs, for each parent and the double pseudo-testcross mapping strategy (Grattapaglia and Sedoroff 1994). This strategy only allows the detection of allele-substitution effects at each parental genome which is less powerful for QTL detection than the cross-pollinated (CP) model, and too abstract for practical use in breeding programs. Instead of SNPs, using SSR, IRAP and SCAR markers has allowed us the detection of up to the four possible genotypes (ac, ad, bc and bd) segregating in a Citrus $(\mathrm{ab}) \times$ Poncirus $(\mathrm{cd})$ progeny, in some genomic regions, and the use of the $\mathrm{CP}$ model (instead of the pseudo-testcross strategy) resulting in the gain of power in the QTL detection (Van Ooigen 2009). Thus, 16 out of 34 QTLs in Table 2 were not detected using the pseudo-testcross strategy, indicating they would correspond to intralocus interactions in the CP model. This model is more realistic and useful for breeding purposes. Besides, intralocus interactions may be molecularly important in citrus. Thus, Jiao et al. (2013) found that $11.7 \%$ of heterozygous genes in C. sinensis were differentially expressed. Then, it could be reasonable to expect a higher percentage of differentially expressed heterozygous genes in $C$. reshnii $\times$ Poncirus trifoliata hybrids given the large genetic distance between their parental species (Herrero et al. 1996).

Another limitation of the present QTL analysis is the limited extent of integrated or consensus Citrus-Poncirus linkage groups, not due to the progeny size (151) but more likely to the cytogenetic differences that exist between $C$. reshnii and $P$. trifoliata (Barros et al. 2010, Mendes et al. 2011).

Taken together both limitations, the size of the phenotyped progeny and the extent of Citrus-Poncirus linkage groups, they explain the low number of the QTLs detected in this study and the scarcity of common QTLs between treatments (2), although most traits were not globally affected by the treatment (Table 1). In general, more QTLs were detected under low-Fe than control conditions, in agreement with the differences in trait heritabilities between treatments.

Rootstock effects on fruit quality traits and the effect of lowering external $\mathrm{Fe}$ nutrition.

In the case of mandarin fruits, the allocation of minerals (fruit $v s$ leaf) is important regarding human health and nutrition. Results on Clausellina fruit concentration of elements (Table 1) generally agree the ranking reported in previous studies (Lado et al. 2016; Czech et al. 2020; Hong et al. 2018); however, there are two 
remarkable differences: the presence of $\mathrm{Si}$, particularly when using the parents as rootstocks, and the relative amount of Sulphur, the fourth element regarding concentration. These are good news. Sulfur is an essential dietary mineral primarily because amino acids contain it. Sulphur is thus considered fundamentally important to human health, and conditions such as nitrogen imbalance and protein-energy malnutrition may result from its deficiency. The detection of rootstock QTLs controlling the fruit concentration of mineral elements ( $\mathrm{Fe}, \mathrm{K}, \mathrm{Cu}, \mathrm{Na}$ and $\mathrm{S}$ ), maturation index (C_SSC/A), and fruit juice acidity $\left(\mathrm{Fe} \_\mathrm{NaOH}\right)$ in the present experiment (Table 2) supports the hypothesis that rootstock genotype is contributing to the level of some nutrients in the fruit, such as it was also found in tomato (Asins et al. 2020). Therefore, those mandarin quality traits could be improved through rootstock breeding programs.

In general, leaf concentrations of elements are highly correlated between Fe treatments what it is not the case in fruit except for Si (Online Resource 3). Lowering the external iron input significantly affected S3, Fe_F and Fe_F/Lf (Online Resource 2, Fig. 2) suggesting less iron is moved (partitioned) towards the fruit what might be seen as the consequence of a regulatory process induced by the plant sensing. If we reduce this sensitivity through the rootstock, we might be able to maintain crop yield and, at the same time, fruit iron content, lowering fertilization costs. Other changes related to the trait correlations were observed. Under low-Fe, $\mathrm{Fe} \_\mathrm{F}$ is positively related to $\mathrm{Cu} \_\mathrm{F}$, Mn_F, Ca_F, FeUEp and S3 (Online Resource 4, Fig. 4). From these traits, Fe_F was only significantly related to $\mathrm{Mn} \_\mathrm{F}$ and $\mathrm{Ca} \_\mathrm{F}$ under control conditions where it was found negatively related to TDFWp and FNm (Fig. 3). These findings suggest that Fe_F is limited by the total dried pulp weight yielded only under control conditions (the more TDFWp, the less Fe_F), while under low-Fe, the iron fruit concentration appeared as the main trait (lowest p-value) related to the status of photosynthesis machinery (measured by SPAD value, S3). Decreases in S3 with Fe deficiency have been previously observed in different plant species, including fruit trees such as peach and citrus (see Martinez-Cuenca et al. 2013). The parents of the rootstock segregating population were significantly different for S3 (Online Resource 1) under low-Fe where, unexpectedly, Flying Dragon (Poncirus trifoliata) behaved as more tolerant than Cleopatra. P. trifoliata is usually considered more susceptible to iron chlorosis than Cleopatra (Castle 1987). This disagreement in the ranking might be due to differences in the treatment to induce low-Fe availability at the root (rising $\mathrm{pH} v s$ decreasing $[\mathrm{Fe}]$ of 
nutrient solution), and/or intraspecific genetic and agronomic diversity within the species P. trifoliata (Fang et al. 1997, Ben Yahmed et al. 2016, Huang et al. 2018). Two QTLs were detected for S3, only under low-Fe; one of them, on LG $10+5$ b was also detected in the corresponding LG of Cleopatra. Given that both QTLs were epistatic (Figure 5B), this interaction has to be considered to select the best (tolerant) genotype combination (ac at CT19.165, and $\mathbf{l m}$ at C1,1600).

Candidate gene analysis

The causal relationship between variation of an agronomic trait and genotypic differences is important for developing targeted strategies in molecular breeding (Varshney et al. 2014). Therefore, any functional or bioinformatics analysis to allow priorization of candidate genes in QTL regions is valuable to guide further experimentation and validation of causal genes underlying QTLs (Bargsten et al. 2014). The clustering of QTLs involved in rootstock mediated tolerance to iron deficiency and fruit quality traits in five genomic regions led us to investigate their gene contents (assuming collinearity with the $C$. clementina genome in these regions), looking for molecular functions and biological processes that were more frequent than expected (i.e. overrepresented), and putative functional candidates. Thus, the genomic region I containing a QTL for fruit concentration of copper was particularly rich in nutrient reservoir activity. This region contains numerous germin-like proteins and all annotated glutathione gamma-glutamylcysteinyltransferases (Online Resource 6) which are involved in the synthesis of phytochelatins, the heavy-metal-binding peptides of plants (Ramos et al. 2007).

Citrus-Poncirus genomic regions containing clusters II and III were particularly relevant for the total fruit content of $\mathrm{Fe}$ and other nutrients $(\mathrm{S}, \mathrm{P}, \mathrm{Mg})$ under low-Fe stress (Figure 6, Table 2). Among numerous transporters, a metal-nicotianamine transporter YSL3 (Ciclev10019170m) is within cluster II. Yellow Stripe-Like (YSL) family of proteins are transporters of metals that are bound to the metal chelator nicotianamine or the related set of mugineic acid family chelators known as phytosiderophores. In Arabidopsis, AtYSL1 and AtYSL3 are localized to the plasma membrane, function as iron transporters (Chu et al. 2010) and are regulated in response to the Fe status of the plant (Waters et al. 2006). In citrus roots, Fe deficiency promoted the expression of a PAL gene and the accumulation of phenolic compounds what could promote Fe solubilization (Yang et al. 2016). A gene coding for PAL is located in cluster III, and a gene coding for transcription factor MYB 54, required to activate the 
expression of PAL in Arabidopsis (https://www.uniprot.org/uniprot/Q9LK95) is in cluster II. As a result of Fe deficiency, Martinez-Cuenca et al. (2013) found increased citrate and malate concentrations in xylem sap and root exudates of Carrizo citrange and this was concomitant with the differential expression of several enzymes related to their metabolism. Genes coding for glutamate decarboxylase, malate dehydrogenase, pyruvate kinase, and dihydrolipoyllysine-residue acetyltransferase component 1 of pyruvate dehydrogenase complex were found within clusters II and III (Online Resource 6). Besides, a gene coding for phosphoenolpyruvate carboxylase 1 (Ciclev100014164m) is within the QTL interval for Fe_S3 on linkage group 10+5b.

Genes involved in oxidative stress response have been frequently reported to be differentially expressed in citrus roots as a consequence of Fe deficiency (Forner-Giner et al. 2009, Licciardello et al. 2013, Muccilli et al. 2013). Two Fe-Mn superoxide dismutases, several glutathione-S transferases, and a thioredoxin and a ferredoxin (Ferredoxin-Thioredoxin system), were found within cluster III. A great deal of evidence has shown that numerous environmental factors and pathogens can induce ROS generation in plant cells (Huang et al. 2019).

Regarding transcriptional regulation, four genes coding for bHLH transcription factors that could play a relevant role in Fe homeostasis under low-Fe stress in Citrus grandis (Zhang et al. 2020) have been found in QTL regions I, III and V. Two of them, bHLH 137.1 and ILR3 are predicted to interact with PYE, another bHLH factor that is a key regulator of Fe deficiency responses. In Arabidopsis, ILR3 is likely a mobile protein that affects rhizosphere acidification under Fe deficiency and modulates multiple stress responses, including cyst nematode infection (Samira et al. 2018).

A particularly important Citrus-Poncirus genomic region corresponds to QTL cluster IV (Figure 6). It includes QTLs for fruit yield (FN and TFW) under both Fe treatments. Noteworthy, response to brassinosteroid stimulus is a biological process that has been associated with yield (Bargsten et al. 2014), and two genes coding for UDPglucosyl transferase 73C [EC:2.4.1.-] Glc-brassinosteroid (UGT73C) that could inactivate brassinosteroids (Husar et al. 2011) are within this region. A known transcriptional factor, the Ethylene Responsive factor ERF SHN/WIN 1 (Ciclev10027305 in Online Resource 6), that has been suggested as an important candidate for improvement of abiotic stress tolerance in crops (Djemal et al. 2018), is in this region too. It is important to point out that cluster IV region also contains the major QTL governing Citrus Tristeza Virus multiplication (Asins et al. 2012), and two QTLs 
for foliar Huanglongbing symptoms (FS-2015-S7a and FS-2016-S7a, Huang et al.

439 2018). Reductions in Fe and $\mathrm{Zn}$ leaf concentrations have been observed after infection

440 by Candidatus liberribacter asiaticus, the causal organism of Huanglongbing in Asia 441 (Masaoka et al. 2011). This genomic region is enriched in genes related to DNA metabolic process and cellular response to stress (Online Resource 5), and it is rich in disease resistance genes (Online Resource 6). Noteworthy, cluster II is enriched in genes coding for the regulatory protein NPR1 that is a key regulator of the SA-mediated SAR pathway that mediates cross-talk between salicylic acid and jasmonic/ethylene responses (Backer et al. 2019), and regulates SA-mediated expression of the metal transporter YSL3 (Chen et al. 2014). Cluster II is also rich in AIG1 domain-containing proteins. This domain is related to resistance against bacteria (https://pfam.xfam.org/family/AIG1). Differential proteins related to the plant defense were previously reported when comparing root protein profiles of two citrus rootstocks (low-Fe tolerant and sensitive) under Fe deficiency conditions (Muccilli et al. 2013).

In conclusion, for first time, a genetic analysis of citrus rootstock-mediated tolerance to iron deficiency and total iron fruit content has been carried out unveiling four genomic regions involved in natural genetic variation for those traits, likely harboring candidate genes that deserve future research to assess their final degree of responsibility in explaining total variability in order to be used in molecular breeding programs of citrus rootstocks. A genetic connection between citrus rootstock-mediated tolerance to Fe deficiency and biotic stresses, based on genes within QTL regions II, IV and $\mathrm{V}$, has been found.

\section{Acknowledgments}

We thank Mrs. Miryam Rojas at Servicio de Instrumentación Científica de la Estación Experimental del Zaidín (CSIC) for mineral analysis and Mr. José Cerdá for technical assistance. This work was supported by grants from the Spanish Government (MJA) (AGL2014-56675-R, AGL2017-82452-C2-2-R),

\section{Data archiving statement}

The SSR primer sequences are available upon request, for scientific purposes only, from the corresponding author mjasins@ivia.es. The genetic linkage maps were submitted to the Citrus Genome Database (https://www.citrusgenomedb.org/). Other markers are described in Raga et al. (2016). The parents of the progeny are kept at the Citrus Germplasm Bank, and the accession references are: IVIA-385 (Cleopatra mandarin), IVIA-537 (Flying Dragon trifoliate orange) and IVIA-236 (Rich trifoliate 
orange). Genomic data on candidate genes are provided as electronic supplementary material EMS_6.

Conflict of interest: The authors declare that they have no conflict of interest.

Ethical standards: The authors declare that the experiment complies with the

current laws.

\section{References}

Abadía J, Alvarez-Fernandez A, Rombolà AD, Sanz M, Tagliavini M, Abadía M (2004) Technologies for the diagnosis and remediation of Fe deficiency. Soil Sci Plant Nutr 50:965-971

Almaliotis DD, Manganaris AG, Simonis AD, Bladenopoulou SB (1995) Rootstock effect on yield and mineral nutrition of 'Maycrest? Peach trees under conditions of lime-induced chlorosis. In: Abadía J (Ed.) Iron nutrition in soils and plants. Kluwer Academic Publishers, Dordrecht, pp 301-306

Asins MJ, Fernández-Ribacoba J, Bernet GP, Gadea J, Cambra M, Gorris MT, Carbonell EA (2012) The position of the major QTL for Citrus tristeza virus resistance is conserved among Citrus grandis, $C$. aurantium and Poncirus trifoliata. Molecular Breeding 29:575-587

Asins MJ, Raga MV, Torrent D, Roca D, Carbonell EA (2020) QTL and candidate gene analyses of rootstock-mediated tomato fruit yield and quality traits under low iron stress. Euphytica. https://doi.org/10.1007/s10681-020-02599-6

Backer R, Sanushka Naidoo S, van den Berg N (2019) The NONEXPRESSOR OF PATHOGENESIS-RELATED GENES 1 (NPR1) and related family: mechanistic insights in plant disease resistance. Front Plant Sci 10:102. https://doi.org/10.3389/fpls.2019.00102

Bargsten JW, Nap J-P, Sanchez-Perez GF, van Dijk ADJ (2014) Priorization of candidate genes in QTL regions based on associations between traits and biological processes. BMC Plant Biol 14:330

Barros e Silva AE, Marques A, dos Santos KGB, Guerra M (2010) The evolution of CMA bands in Citrus and related genera. Chromosome Res 18:503-5014

Ben Yahmed J, Costantino G, Amiel P, Talon M, Ollitrault P, Morillon R, Luro F (2016) Diversity in the trifoliate orange taxon reveals two main genetic groups marked by specific morphological traits and water deficit tolerance properties. J Agric Sci 154:495-514. https://doi.org/10.1017/S0021859615000234 
505

506

507

508

509

510

511

512

513

514

515

516

517

518

519

520

521

522

523

524

525

526

527

528

529

530

531

532

533

534

535

536

Castle W S, Rom RC, Carlson RF (1987) Rootstocks for fruit crops. Citrus rootstocks. Wiley, New York, p. 361-399

Castle WS, Nunnallee J, Manthey JA (2009.) Screening Citrus Rootstocks and Related Selections in Soil and Solution Culture for Tolerance to Low-iron Stress. Hortscience 44: 638-645

Chen C-C, Chien W-F, Lin N-C, Yeh K-C (2014) Alternative functions of Arabidopsis YELLOW STRIPE-LIKE3: from metal translocation to pathogen defense. PLOS ONE 9: e98008. https://doi.org/10.1371/journal.pone.0098008

Chu H-H, Chiecko J, Punshon T, Lanzirotti A, Lahner B, Salt DE, Walker EL (2010) Successful Reproduction Requires the Function of Arabidopsis YELLOW STRIPE-LIKE1 and YELLOW STRIPE-LIKE3 metal-nicotianamine transporters in both vegetative and reproductive structures. Plant Physiol 154:197-210

Czech A, Zarycka E, Yanovych D, Zasadna Z, Grzegorczyk I, Klys S, (2020) Mineral content of the pulp and peel of various citrus fruit cultivars. Biol Trace Elem Res 193:555-563. https://doi.org/10.1007/s12011-019-01727-1

Djemal R, Mila I, Bouzayen M, Pirrello J, Khoudi H (2018) Molecular cloning and characterization of novel WIN1/SHN1 ethylene responsive transcription factor HvSHN1 in barley (Hordeum vulgare L.). J Plant Physiol 228:39-46. https://doi: 10.1016/j.jplph.2018.04.019.

Fang, D. Q., Roose, M. L., Krueger, R. R. \& Federici, C. T. (1997). Fingerprinting trifoliate orange germ plasm accessions with isozymes, RFLPs, and inter-simple sequence repeat markers. Theor Appl Genet 95:211-219.

Fernandez V, Winkelmann G, Ebert G (2004) Iron supply to tobacco plants though foliar application of iron citrate and ferric dimerum acid. Physiol Plant 122:380385

Forner-Giner MA, Llosa MJ, Carrasco JL, Perez-Amador MA, Navarro L, Ancillo G (2009) Differential gene expression analysis provides new insights into the molecular basis of iron deficiency stress response in the citrus rootstock Poncirus trifoliata (L.) Raf. J Exp Bot 61:483-490. https://doi.org/10.1093/jxb/erp328

Fu LN, Zhu QQ, Sun YY, Du W, Pan ZY, Peng SA (2017) Physiological and Transcriptional Changes of Three Citrus Rootstock Seedlings under Iron Deficiency. Front Plant Sci 8: 1104. https://doi.org/10.3389/fpls.2017.01104 
Grattapaglia D, Sederoff RR (1994) Genetic linkage maps of Eucaliptus grandis and E. urophylla using a pseudo-testcross mapping strategy and RAPD markers. Genetics 137:1121-1137

Herrero R, Asins MJ, Carbonell EA, Navarro L (1996) Genetic diversity in the orange subfamily Aurantioideae. II. Genetic relationships among genera and species. Theor Appl Genet 93:1327-1334

Hong YS, Choi JY, Nho EY, Hwang IM, Khan N, Jamila N, Kim KS (2019) Determination of macro, micro and trace elements in citrus fruits by inductively coupled plasma-optical emission spectrometry (ICP-OES), ICP-mass spectrometry and direct mercury analyzer J. Sci. Food Agric 99:1870-1879. https://doi.org/10.1002/jsfa.9382

Huang H, Ullah F, Zhou D-X, Yi M, Zhao Y (2019) Mechanisms of ROS regulation of plant development and stress responses. Front Plant Sci 10:800. https://doi.org/10.3389/fpls.2019.00800

Huang M, Roose ML, Yu Q, Du D, Yu Y, Zhang Y, Deng Z, Stover E, Gmitter FG (2018) Construction of high-density genetic mapas and detection of QTLs associated with Huanglongbing tolerance in citrus. Front Plant Sci 9:1694 https://doi.org/10.3389/fpls.2018.01694

Husar S, Berthiller F, Fujioka S, Rozhon W, Khan M, Kalaivanan F, Elias L, Higgins GS, Li Y, Schuhmacher R, Krska R, Seto H, Vaistij FE, Bowles D, Poppenberger B. (2011) Overexpression of the UGT73C6 alters brassinosteroid glucoside formation in Arabidopsis thaliana. BMC Plant Biol 11:51. https://doi.org/10.1186/1471-2229-11-51.

Jaegger B, Goldbach H, Sommer K (2000) Release from lime induced iron chlorosis by CULTAN in fruit trees and its characterization by analysis Acta Hort 531:107-113 Jessop RS, Roth G, Sale P (1990) Effects of increased levels of soil $\mathrm{CaCO}_{3}$ on lupin (Lupinus angustifolius) growth and nodulation. Aust J Soil Res 28:955-962

Jiao W-B, Huang D, Xing F, Hu Y, Deng X-X, Xu Q, Chen L-L (2013) Genome-wide characterization and expression analysis of genetic variants in sweet orange. The Plant Journal 75:954-964

Korcak R (1987) Iron deficiency chlorosis. Hort Rev (Amer Soc Hort Sci) 9:133-186

Lado J, Cuellar F, Rodrigo MJ, Zacarias L (2016) Nutritional Composition of Mandarins. In: Simmonds MSJ, Preedy VR (eds) Nutritional composition of fruit 
cultivars Elsevier Inc pp: 419-443. https://doi.org/10.1016/B978-0-12-408117$8.00018-0$

Licciardello C, Torrisi B, Allegra M, Sciacca F, Roccuzzo G, Intrigliolo F, Recupero GR, Tononi P, Delledonne M, Muccilli V (2013) A Transcriptomic Analysis of Sensitive and Tolerant Citrus Rootstocks under Natural Iron Deficiency Conditions. J Am Soc Hortic Sci 138: 487-498

Lima RPM, Curtolo M, Merfa MV, Cristofani-Yali M, Machado MA (2018) QTLs and eQTLs mapping related to citrandarins resistance to citrus gummosis disease. BMC Genomics 19:516 https://doi.org/10.1186/s12864-018-4888-2

Manthey JA, McCoy DL, Crowley DE (1994) Stimulation of rhizosphere iron reduction and uptake in response to iron deficiency in citrus rootstocks. Plant Physiol Biochem 32:211-215

Martinez-Cuenca MR, Iglesias DJ, Talon M, Abadia J, Lopez-Millan AF, Primo-Millo E, Legaz F (2013) Metabolic responses to iron deficiency in roots of Carrizo citrange [Citrus sinensis (L.) Osbeck. x Poncirus trifoliata (L.) Raf.] Tree Physiol 33:320-329. https://doi.org/10.1093/treephys/tpt011

Masaoka Y, Pustika A, Subandiyah S, Okada A, Hanundin E, Purwanto B, Okuda M, Okada Y, Saito A, Holford P, Beattie A, Iwanami T (2011) Lower concentrations of microelements in leaves of citrus infected with Candidatus Liberibacter asiaticus. Jarq-Jpn Agr Res Q 45:269-275. https://doi.org/10.6090/jarq.45.269

Mendes S, Moraes AP, Mirkov TE, Pedrosa-Harand A (2011) Chromosome homeologies and high variation in heterochromatin distribution between Citrus L. and Poncirus Raf. as evidenced by comparative cytogenetic mapping. Chromosome Res 19: 521-530 HTTPS://DOI.ORG/10.1007/s10577-011-9203-x

Mengel K (1994) Iron availability in plant-tissues-iron chlorosis on calcareous soils. Plant Soil 165: 275-283

Muccilli V, Licciardello C, Fontanini D, Cunsolo V, Capocchi A, Saletti R, Torrisi B, Foti S (2013) Root protein profiles of two citrus rootstocks grown under iron sufficiency/deficiency conditions. Eur J Mass Spectrom 19:305-324. https://doi.org/10.1255/ejms.1230

Pestana M, de Varennes A, Abadia J, Faria EA (2005) Differential tolerance to iron deficiency of citrus rootstocks grown in nutrient solution. Scientia Horticulturae 104: 25-36 HTTPS://DOI.ORG/10.1016/j.scienta.2004.07.007 
Pestana M, Correia PJ, David M, Abadia A, Abadia J, de Varennes, A (2011) Response of five citrus rootstocks to iron deficiency. J Soil Sci Plant Nutr 174:837-846. https://doi.org/10.1002/jpln.201000341

Raga V, Bernet GP, Carbonell EA, Asins MJ (2012) Segregation and linkage analyses in two complex populations derived from the citrus rootstock Cleopatra mandarin. Inheritance of seed reproductive traits. Tree Genetics and Genomes 8:1061-1071

Raga V, Intrigliolo DS, Bernet GP, Carbonell EA and MJ Asíns. 2016. Genetic analysis of salt tolerance in a progeny derived from the citrus rootstocks Cleopatra mandarin and trifoliate orange. Tree Genetics and Genomes 12:34. https://doi.org/10.1007/s11295-016-0991-1

Ramos J, Clemente MR, Naya L, Loscos J, Perez-Rontome C, Sato S, Tabata S, Becana M (2007) Phytochelatin synthases of the model legume Lotus japonicus. A small multigene family with differential response to cadmium and alternatively spliced variants. Plant Physiol 143:1110-1118

Rashid A, Ryan J (2004) Micronutrient constraints to crop production in soils with Mediterranean-type characteristics: A review. J Plant Nutr 27:959-975

Ruiz C, Bretó MP, Asins MJ (2000) An efficient methodology to identify sexual seedlings in citrus breeding programs using SSR markers. Euphytica 112:89-94.

Samira R, Li B, Kliebenstein D, Li C, Davis E, Gillikin JW, Long TA(2018) The bHLH transcription factor ILR3 modulates multiple stress responses in Arabidopsis. Plant Molecular Biology (2018) 97:297-309. https://doi.org/10.1007/s11103-018-07358

Tian T, Liu Y, Yan H, You Q, Yi X, Du Z, Xu W, Su Z (2017) agriGO v2.0: a GO analysis toolkit for the agricultural community, update. Nucleic Acids Res 45:W122-W129. https://doi.org/10.1093/nar/gkx382

Van Ooijen JW (2009) MapQTL 6 software for the mapping of quantitative trait loci in experimental populations of diploid species. Kyazma, Wageningen, The Netherlands

Van Ooijen JW (2012) JoinMap 4.1 software for the calculation of genetic linkage maps in experimental populations. Kyazma, Wageningen, The Netherlands

Varshney RK, Terauchi R, McCouch SR (2014) Harvesting the promising fruits of genomics: applying genome sequencing technologies to crop breeding. PLoS Biol 12:e1001883 
Villalta I, Bernet GP, Carbonell EA, Asins MJ (2007) Comparative QTL analysis of salinity tolerance in terms of fruit yield using two Solanum populations of $\mathrm{F}_{7}$ lines. Theor Appl Genet 114:1001-1017

Waters BM, Chu HH, Didonato RJ, Roberts LA, Eisley RB, Lahner B, Salt DE, Walker EL (2006) Mutations in Arabidopsis Yellow Stripe-Like1 and Yellow Stripe-Like3 reveal their roles in metal ion homeostasis and loading of metal ions in seeds. Plant Physiol 141: 1446-1458

Yang HY, Dong T, Li JF, Wang MY (2016) Molecular cloning, expression, and subcellular localization of a PAL gene from Citrus reticulata under iron deficiency. Biol Plantarum 60:482-488. https://doi.org/10.1007/s10535-016-06253

Zhang X-Y, Qiu J-Y, Hui Q-L, Xu Y-Y, He Y-Z, Peng L-Z, Fu X-Z (2020) Systematic analysis of the basic/helix-loop-helix (bHLH) transcription factor family in pummelo (Citrus grandis) and identification of the key members involved in the response to iron deficiency. BMC genomics 21(1): 233. https://doi.org/10.1186/s12864-020-6644-7

Wu, G., Terol, J., Ibanez, V. et al. (2018) Genomics of the origin and evolution of Citrus. Nature 554, 311-316. https://doi.org/10.1038/nature25447 
Table 1- The mean (and standard error) of the phenotypic values for the analyzed traits in parents (Cleopatra, Cleo, and Flying Dragon, Pon) and minimum (Min) and

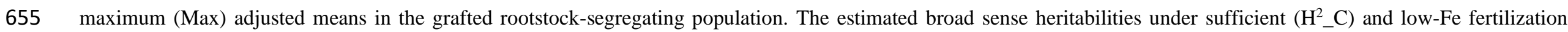

$656\left(\mathrm{H}^{2} \_\mathrm{Fe}\right)$ are also included. Not analysed is denoted by na.

\begin{tabular}{|c|c|c|c|c|c|c|c|c|c|c|c|}
\hline Abbrev. & TRAIT & Cleo_C & Pon_C & Cleo_Fe & Pon_Fe & min_C & max_C & min_Fe & max_Fe & H2_C & H2_Fe \\
\hline Al_F & [Al] in fruit & $1.77 \pm 0.27$ & $1.53 \pm 0.01$ & $6.96 \pm 4.9$ & $2.46 \pm 0.18$ & 1.33 & 12.91 & 1.39 & 15.60 & 0.0000 & 0.0000 \\
\hline Ca_F & {$[\mathrm{Ca}]$ in fruit } & $3116.64 \pm 74.75$ & $2621.54 \pm 51.81$ & $4978.22 \pm 912.02$ & $2609.89 \pm 462.22$ & 1531.75 & 5371.76 & 1357.26 & 4814.08 & 0.0109 & 0.0000 \\
\hline $\mathbf{C u} \mathbf{C F}_{\mathbf{F}}$ & {$[\mathrm{Cu}]$ in fruit } & $6.36 \pm 0.31$ & $6.87 \pm 1.02$ & $4.27 \pm 0.53$ & $5.54 \pm 0.75$ & 3.15 & 8.51 & 3.52 & 8.98 & 0.0031 & 0.2427 \\
\hline $\mathbf{F e} \_\mathbf{F}$ & {$[\mathrm{Fe}]$ in fruit } & $25.15 \pm 1.46$ & $20.42 \pm 2.05$ & $18.88 \pm 0.89$ & $22.94 \pm 2.27$ & 19.32 & 45.32 & 14.35 & 31.55 & 0.0333 & 0.0000 \\
\hline K_F & {$[\mathrm{K}]$ in fruit } & $16231.33 \pm 12.12$ & $14135.5 \pm 612.26$ & $14592.02 \pm 958.53$ & $15350.2 \pm 964.58$ & 9466.87 & 17660.35 & 9715.66 & 17705.60 & 0.0064 & 0.4726 \\
\hline Mg_F & {$[\mathrm{Mg}]$ in fruit } & $1261.8 \pm 4.57$ & $942.91 \pm 59.69$ & $1157.95 \pm 93.11$ & $1035.36 \pm 9$ & 823.92 & 1276.85 & 817.77 & 1409.93 & 0.2316 & 0.2659 \\
\hline Na_F & {$[\mathrm{Na}]$ in fruit } & $88.3 \pm 21.56$ & $91.6 \pm 47.24$ & $336.46 \pm 36.54$ & $74.68 \pm 0.19$ & -90.05 & 395.26 & -15.79 & 734.16 & 0.0000 & 0.0738 \\
\hline P_F & {$[\mathrm{P}]$ in fruit } & $3561.01 \pm 34.49$ & $3202.36 \pm 156.58$ & $3250.25 \pm 241.98$ & $3527.71 \pm 26.38$ & 1978.95 & 3881.67 & 2175.17 & 4163.97 & 0.0000 & 0.1938 \\
\hline S_F & {$[S]$ in fruit } & $1955.32 \pm 58.89$ & $1642.13 \pm 13.48$ & $1947.28 \pm 166.25$ & $1687.36 \pm 33.56$ & 1238.23 & 1940.98 & 1278.30 & 2281.79 & 0.1192 & 0.1419 \\
\hline Si_F & [Si] in fruit & $18.12 \pm 0.08$ & $14.6 \pm 2.5$ & $20.14 \pm 5.04$ & $19.32 \pm 0.64$ & -0.74 & 22.29 & -0.23 & 21.67 & 0.3570 & 0.4561 \\
\hline Zn_F & {$[\mathrm{Zn}]$ in fruit } & $30.51 \pm 0.58$ & $33.4 \pm 2.12$ & $44.11 \pm 19.47$ & $32.02 \pm 7.51$ & 9.83 & 64.23 & 10.28 & 47.91 & 0.0000 & 0.0000 \\
\hline Al_Lf & [Al] in leaf & $94.94 \pm 6.05$ & $64.91 \pm 21.81$ & $119.38 \pm 5.19$ & $97.79 \pm 37.7$ & 20.48 & 123.31 & 23.95 & 156.40 & 0.5373 & 0.4002 \\
\hline Cu_Lf & {$[\mathrm{Cu}]$ in leaf } & $11.55 \pm 3.12$ & $8.95 \pm 2.7$ & $6.32 \pm 2.11$ & $16.11 \pm 13.06$ & 2.82 & 29.66 & 4.19 & 34.31 & 0.0315 & 0.0000 \\
\hline Fe_Lf & {$[\mathrm{Fe}]$ in leaf } & $50.76 \pm 3.37$ & $64.5 \pm 9.42$ & $52.05 \pm 7.12$ & $68.49 \pm 0.15$ & 37.69 & 76.13 & 36.61 & 80.83 & 0.0157 & 0.2205 \\
\hline K_Lf & {$[\mathrm{K}]$ in leaf } & $15122 \pm 534.81$ & $18161.53 \pm 1202.05$ & $16223.77 \pm 1354.4$ & $16237.09 \pm 1034.79$ & 13600.02 & 21174.90 & 11337.82 & 21488.85 & 0.0000 & 0.0000 \\
\hline Mg_Lf & {$[\mathrm{Mg}]$ in leaf } & $1421.42 \pm 289.88$ & $1362.5 \pm 152.43$ & $2716.25 \pm 232.23$ & $1338.99 \pm 459.64$ & 970.84 & 3167.60 & 932.14 & 3376.77 & 0.1869 & 0.1786 \\
\hline Mn_Lf & {$[\mathrm{Mn}]$ in leaf } & $57.52 \pm 5.34$ & $25.65 \pm 1.57$ & $18.44 \pm 0.3$ & $32 \pm 6.67$ & 18.46 & 65.92 & 13.60 & 63.00 & 0.2324 & 0.1181 \\
\hline Na_Lf & {$[\mathrm{Na}]$ in leaf } & $589.12 \pm 24.02$ & $362.43 \pm 165.8$ & $610.11 \pm 85.22$ & $257.02 \pm 53.11$ & 151.32 & 1427.97 & 192.53 & 1988.62 & 0.0239 & 0.2484 \\
\hline P_Lf & {$[\mathrm{P}]$ in leaf } & $2080.33 \pm 492$ & $2545.97 \pm 451.34$ & $1862.71 \pm 69.85$ & $2126.01 \pm 2.99$ & 1319.72 & 3106.53 & 1046.06 & 3690.31 & 0.0511 & 0.0000 \\
\hline S_Lf & {$[S]$ in leaf } & $2714.06 \pm 132.84$ & $2730.46 \pm 35.63$ & $2527.08 \pm 284.97$ & $2493.17 \pm 131.43$ & 1797.83 & 3304.76 & 1717.68 & 3454.48 & 0.0442 & 0.0474 \\
\hline
\end{tabular}




\begin{tabular}{|c|c|c|c|c|c|c|c|c|c|c|c|}
\hline Si_Lf & [Si] in leaf & $307.84 \pm 61.65$ & $170.7 \pm 11.02$ & $354.18 \pm 41.38$ & $245.46 \pm 63.9$ & 122.87 & 361.80 & 146.20 & 438.60 & 0.3886 & 0.0709 \\
\hline Zn_Lf & {$[\mathrm{Zn}]$ in leaf } & $36.99 \pm 11.53$ & $35.23 \pm 6.63$ & $30.84 \pm 8.3$ & $24.63 \pm 1.25$ & 9.59 & 60.39 & 13.93 & 41.93 & 0.1048 & 0.0147 \\
\hline Fe_F_Lf & $100(\mathrm{Fe}$ F/Fe_Lf $)$ & $0.33 \pm 0.03$ & $0.24 \pm 0.01$ & $0.27 \pm 0.02$ & $0.25 \pm 0.02$ & 0.25 & 0.46 & 0.18 & 0.40 & 0.0645 & 0.0000 \\
\hline Al_F_Lf & 100 (Al_F/Al_Lf) & $0.02 \pm 0.0039$ & $0.03 \pm 0.01$ & $0.05 \pm 0.03$ & $0.03 \pm 0.01$ & 0.02 & 0.25 & 0.01 & 0.28 & 0.0000 & 0.0000 \\
\hline LDM & Leaf dry matter & $39.9 \pm 2.4$ & $37.82 \pm 4.49$ & $39.85 \pm 3.01$ & $41.88 \pm 0.97$ & 35.61 & 48.76 & 38.35 & 46.91 & 0.0000 & 0.0000 \\
\hline LFW & Leaf fresh weight & $2.1 \pm 0.5$ & $2.35 \pm 0.25$ & $2.35 \pm 0.45$ & $2.15 \pm 0.05$ & 1.70 & 4.00 & 1.70 & 3.65 & 0.1757 & 0.0000 \\
\hline LDW & Leaf dry weight & $0.85 \pm 0.25$ & $0.9 \pm 0.2$ & $0.95 \pm 0.25$ & $0.9 \pm 0$ & 0.77 & 1.88 & 0.68 & 1.62 & 0.1574 & 0.0000 \\
\hline S3 & SPAD at the end & $77.75 \pm 4.95$ & $69.3 \pm 1$ & $49.25 \pm 18.85$ & $73.9 \pm 1.7$ & 65.22 & 85.38 & 61.27 & 80.78 & 0.1431 & 0.1187 \\
\hline LA & Leaf area & $19.8 \pm 1.9$ & $27.8 \pm 1.6$ & $25.85 \pm 1.15$ & $18.7 \pm 0.4$ & 18.27 & 31.67 & 17.52 & 30.03 & 0.0647 & 0.0000 \\
\hline FNm & Fruit number & $8.5 \pm 0.5$ & $5.5 \pm 1.5$ & $12 \pm 0$ & $7.5 \pm 2.5$ & 0.93 & 23.41 & 1.99 & 20.89 & 0.0248 & 0.0843 \\
\hline TFWm & Total fruit weight & $0.55 \pm 0.01$ & $0.47 \pm 0.03$ & $0.66 \pm 0.14$ & $0.37 \pm 0.13$ & 0.13 & 1.40 & 0.08 & 1.35 & 0.1787 & 0.1770 \\
\hline TDFWp & Total pulp dry weight & $7.76 \pm 0.49$ & $6.31 \pm 1.07$ & $6.19 \pm 0.54$ & $4.22 \pm 0.67$ & 1.24 & 23.59 & 3.35 & 19.40 & 0.0655 & 0.1421 \\
\hline FeUEp & Total harvested Fe & $0.2 \pm 0.02$ & $0.13 \pm 0.01$ & $0.12 \pm 0.0048$ & $0.1 \pm 0.02$ & 0.08 & 0.45 & 0.06 & 0.47 & 0.1376 & 0.3973 \\
\hline PUEp & Total harvested $\mathrm{P}$ & $27.66 \pm 2.01$ & $20.38 \pm 4.41$ & $19.99 \pm 0.27$ & $14.89 \pm 2.46$ & -1.02 & 81.90 & 10.18 & 57.51 & 0.0000 & 0.3082 \\
\hline SUEp & Total harvested Fe & $15.21 \pm 1.42$ & $10.35 \pm 1.67$ & $11.97 \pm 0.03$ & $7.09 \pm 0.98$ & 2.04 & 43.72 & 5.52 & 33.31 & 0.0643 & 0.3623 \\
\hline FW & Fruit weight & $57.27 \pm 7.13$ & $85.69 \pm 14.93$ & $52.84 \pm 10.02$ & $49.87 \pm 3.31$ & 33.64 & 143.94 & 45.58 & 110.80 & 0.0000 & 0.0000 \\
\hline FD & Fruit diameter & $49.36 \pm 1.21$ & $57.61 \pm 3.89$ & $48.5 \pm 3.21$ & $47.74 \pm 0.54$ & 41.00 & 66.50 & 46.50 & 61.88 & 0.0000 & 0.0000 \\
\hline RT & Rind thickness & $1.93 \pm 0.07$ & $2.64 \pm 0.36$ & $1.5 \pm 0.07$ & $1.36 \pm 0.36$ & 1.29 & 4.00 & 1.20 & 3.29 & 0.0000 & 0.0000 \\
\hline JV & Juice volume & $24.21 \pm 2.93$ & $30.71 \pm 2.29$ & $26.57 \pm 3.71$ & $22.91 \pm 1.51$ & 14.64 & 51.75 & 19.61 & 52.54 & 0.0000 & 0.0000 \\
\hline $\mathrm{JC}$ & Juice content & $43.18 \pm 0.03$ & $36.5 \pm 3.79$ & $51.51 \pm 2.59$ & $46.74 \pm 1.26$ & 23.63 & 56.07 & 35.59 & 55.43 & 0.0732 & 0.0000 \\
\hline SSC & Soluble-solids content & $8.2 \pm 0.57$ & $7.65 \pm 0.4$ & $8.14 \pm 1.31$ & $7.55 \pm 0.43$ & 6.63 & 10.48 & 6.31 & 10.83 & 0.0000 & 0.0060 \\
\hline $\mathrm{NaOH}$ & $\mathrm{NaOH}$ volume & $7.8 \pm 0.04$ & $7.69 \pm 0.46$ & $8.26 \pm 2.1$ & $7.81 \pm 0.53$ & 5.69 & 13.14 & 5.94 & 13.38 & 0.0000 & 0.2580 \\
\hline SSC_A & SSC/Acidity ratio & $8.32 \pm 0.51$ & $7.92 \pm 0.03$ & $8.67 \pm 3.38$ & $7.64 \pm 0.04$ & 5.29 & 11.18 & 5.70 & 9.05 & 0.1311 & 0.0000 \\
\hline dAl & change in leaf [Al] & $68.86 \pm 0.54$ & $48.81 \pm 25.85$ & $96.98 \pm 1.63$ & $63.64 \pm 19.09$ & 28.83 & 99.76 & -57.48 & 117.96 & na & 0.2091 \\
\hline dCa & change in leaf [Ca] & $10787.41 \pm 5157.9$ & $7809.84 \pm 3449.66$ & $6360.48 \pm 830.38$ & $8451.16 \pm 5022.27$ & -1791.34 & 14021.09 & -13547.02 & 27496.93 & na & 0.0880 \\
\hline
\end{tabular}




\begin{tabular}{|c|c|c|c|c|c|c|c|c|c|c|c|}
\hline $\mathrm{dCu}$ & change in leaf $[\mathrm{Cu}]$ & $-63.43 \pm 26.07$ & $-42.05 \pm 17.96$ & $-41.29 \pm 6$ & $-37.28 \pm 9.02$ & -57.88 & -7.64 & -53.57 & 0.87 & na & 0.1788 \\
\hline $\mathrm{dFe}$ & change in leaf $[\mathrm{Fe}]$ & $5.71 \pm 1.33$ & $17.23 \pm 6.53$ & $18.17 \pm 6.52$ & $10.7 \pm 0.56$ & -2.82 & 19.17 & -35.19 & 40.23 & na & 0.1336 \\
\hline dK & change in leaf [K] & $-925.46 \pm 725.38$ & $358.67 \pm 2180.94$ & $2878.82 \pm 1858.36$ & $355.45 \pm 1106.42$ & -2041.60 & 11093.08 & -2564.16 & 15736.99 & na & 0.1181 \\
\hline $\mathrm{dMg}$ & change in leaf $[\mathrm{Mg}]$ & $421.67 \pm 245.39$ & $604.29 \pm 116.98$ & $1064.78 \pm 188.44$ & $450.81 \pm 387.82$ & -530.23 & 792.56 & -1168.86 & 2273.86 & na & 0.0692 \\
\hline dMn & change in leaf $[\mathrm{Mn}]$ & $15.88 \pm 0.09$ & $2.26 \pm 7.05$ & $-6.9 \pm 2.2$ & $-0.66 \pm 3.96$ & -14.53 & 28.00 & -12.64 & 27.35 & na & 0.0000 \\
\hline $\mathrm{dNa}$ & change in leaf [Na] & $-65.78 \pm 316.67$ & $-243.79 \pm 241.26$ & $92.42 \pm 26.44$ & $53.82 \pm 14.05$ & -520.42 & 25.70 & -470.62 & 1745.23 & na & 0.2482 \\
\hline dP & change in leaf $[\mathrm{P}]$ & $-780.79 \pm 142.5$ & $274.05 \pm 28.75$ & $241.76 \pm 230.3$ & $-619.34 \pm 439.22$ & -700.47 & 1040.59 & -671.80 & 1738.10 & na & 0.0747 \\
\hline dSi & change in leaf [Si] & $183.19 \pm 36.02$ & $88.53 \pm 21.36$ & $98.74 \pm 10.13$ & $119.36 \pm 40.81$ & -31.48 & 148.01 & -109.25 & 266.64 & na & 0.1581 \\
\hline $\mathrm{dZn}$ & change in leaf [Zn] & $-0.03 \pm 13.44$ & $-13.84 \pm 10.81$ & $-8.15 \pm 0.95$ & $3.26 \pm 3.15$ & -4.66 & 20.86 & -23.67 & 25.72 & na & 0.0000 \\
\hline
\end{tabular}


657 Table 2- List of the position (in cM), LOD, and nearest marker (Locus) to QTLs detected by IM and

658 MQM in the integrated Citrus reshni-Poncirus trifoliata genetic linkage map (LG) using the cross-

659 pollinated model. Those QTL that were also detected at the individual parental linkage maps are indicated

660 by adding the parental linkage group between parenthesis ( $\mathrm{R}$ or $\operatorname{Pr}$ for $C$. reshni and P. trifoliata,

661 respectively). The four genotypic means (ac, ad, bc, and bd, being C. reshni ab and P. trifoliata cd), the

662 percentage of explained variance, PEV, and the genomic region containing QTLs for target traits

663 (Region) are included. These regions, named in Latin numbers (from I to V), were anchored to the $C$.

664 clementina physical map, and the corresponding scaffold number is indicated between parentheses.

\begin{tabular}{|c|c|c|c|c|c|c|c|c|c|c|}
\hline Region & Trait & Group & Position & Locus & LOD & ac & ad & bc & bd & PEV \\
\hline & C_Ca_Lf & $4 c(R)$ & 0.0 & $15 \mathrm{R}, 750$ & 3.1 & 20032.60 & 27005.10 & 27607.90 & 28230.50 & 20.6 \\
\hline & C_Fe_F & $7(\operatorname{Pr})$ & 104.3 & C11iC1rt,400 & 6.6 & 24.99 & 27.97 & 24.34 & 34.24 & 38.9 \\
\hline \multirow[t]{7}{*}{ IV (7) } & C_FNm & $4 \mathrm{~b}$ & 104.8 & Py65C,506 & 3.7 & 8.59 & 16.09 & 13.84 & 2.95 & 24.1 \\
\hline & C_K_F & $4 \mathrm{c}$ & 0.0 & $15 \mathrm{R}, 750$ & 2.5 & 13749.50 & 13721.30 & 12261.60 & 14357.20 & 16.8 \\
\hline & C_Mg_F & $3 \mathrm{a}$ & 20.0 & CR31,100 & 2.8 & 1220.37 & 998.07 & 1033.27 & 1112.23 & 18.9 \\
\hline & C_Si_Lf & 7 & 228.8 & CAC23,230 & 3.3 & 186.04 & 308.56 & 215.12 & 222.71 & 21.4 \\
\hline & C_SSC/A & $3 b(R)$ & 8.9 & C8iC1rt,650 & 2.5 & 7.53 & 7.08 & 8.30 & 7.92 & 16.9 \\
\hline & C_SSC/A & $4 \mathrm{~b}(\mathrm{Pr})$ & 54.4 & CR3,320 & 3.7 & 8.83 & 7.42 & 7.90 & 6.91 & 24.4 \\
\hline & C_SUEp & 2 & 64.0 & TAA1,180 & 3.0 & 76.50 & -16.64 & -27.37 & 33.32 & 20.4 \\
\hline IV (7) & C_tFWm & $4 \mathrm{~b}$ & 104.8 & Py65C,506 & 3.8 & 0.64 & 1.01 & 0.85 & 0.27 & 24.6 \\
\hline \multirow[t]{3}{*}{ I (3) } & $\mathrm{Fe} \_\mathrm{Cu} \_\mathrm{F}$ & 7 & 71.4 & CR17,300 & 4.9 & 6.79 & 5.51 & 5.37 & 6.67 & 29.4 \\
\hline & $\mathrm{Fe} \_\mathrm{Cu} \_\mathrm{F}$ & $3 \mathrm{~b}$ & 9.9 & $\mathrm{C} 8 \mathrm{iC} 1 \mathrm{rt}, 650$ & 3.2 & 6.79 & 7.34 & 6.40 & 5.24 & 18.3 \\
\hline & Fe_dAl & $8(\operatorname{Pr})$ & 214.7 & HD-ZIP,510 & 3.0 & 21.34 & 20.49 & 47.09 & -18.36 & 20.1 \\
\hline $\mathrm{V}(1)$ & $\mathrm{Fe} \_\mathrm{dCu}$ & $3 b$ & 0.0 & $\mathrm{C} 1,1600$ & 2.4 & -27.74 & -39.33 & -38.16 & -17.42 & 16.5 \\
\hline $\mathrm{V}(1)$ & $\mathrm{Fe} \_\mathrm{dK}$ & $3 \mathrm{~b}$ & 0.0 & $\mathrm{C} 1,1600$ & 4.7 & 1889.38 & 2115.62 & 1921.90 & 8871.46 & 29.2 \\
\hline II (3) & Fe_FeUEp & $7(\operatorname{Pr})$ & 138.2 & $\mathrm{C} 1 \mathrm{iC} 8 \mathrm{rt}, 515$ & 5.2 & 0.30 & 0.15 & 0.19 & 0.22 & 31.9 \\
\hline III (4) & Fe_FeUEp & $12(\mathrm{R})$ & 46.8 & CMS20,170 & 3.1 & 0.21 & 0.16 & 0.26 & 0.24 & 20.7 \\
\hline III (4) & Fe_FNm & 12 & 41.9 & $6 \mathrm{~F} 5 \mathrm{R}, 1200$ & 3.2 & 10.19 & 7.79 & 14.64 & 11.48 & 21.4 \\
\hline IV (7) & Fe_FNm & $4 \mathrm{~b}$ & 95.5 & Py65C,506 & 3.2 & 8.26 & 13.69 & 14.21 & 4.43 & 21.3 \\
\hline II (3) & Fe_MgUEp & 7 (Pr) & 137.2 & $\mathrm{C} 1 \mathrm{iC} 8 \mathrm{rt}, 515$ & 4.4 & 13.28 & 7.19 & 9.87 & 9.85 & 27.6 \\
\hline \multirow[t]{4}{*}{ III (4) } & Fe_MgUEp & $12(\mathrm{R})$ & 48.8 & CMS20,170 & 3.9 & 9.37 & 7.40 & 11.92 & 11.70 & 24.9 \\
\hline & Fe_Mn_Lf & 2 & 170.1 & NAC1,520 & 3.2 & 37.59 & 47.33 & 41.75 & 23.84 & 20.8 \\
\hline & $\mathrm{Fe} \_\mathrm{Na} \_\mathrm{F}$ & $4 c(R)$ & 26.8 & CR28,270 & 3.6 & 197.65 & 329.86 & 402.63 & 363.50 & 23.4 \\
\hline & $\mathrm{Fe} \_\mathrm{NaOH}$ & $12(\mathrm{R})$ & 172.9 & $\mathrm{C} 11 \mathrm{iC} 1 \mathrm{rt}, 350$ & 3.0 & 5.09 & 7.71 & 13.53 & 10.33 & 20 \\
\hline II (3) & Fe_PUEp & $7(\operatorname{Pr})$ & 136.2 & $\mathrm{C} 1 \mathrm{iC} 8 \mathrm{rt}, 515$ & 4.5 & 38.42 & 19.10 & 27.03 & 27.36 & 28.4 \\
\hline \multirow[t]{3}{*}{ III (4) } & Fe_PUEp & $12(\mathrm{R})$ & 49.8 & CMS20,170 & 4.1 & 23.84 & 20.29 & 35.38 & 32.43 & 26 \\
\hline & $\mathrm{Fe} \_\mathrm{S} \_\mathrm{F}$ & $8(\mathrm{R} 8+6)$ & 82.0 & EMA_M30 & 4.7 & -1078.06 & 4705.05 & 3253.26 & -514.32 & 32 \\
\hline & Fe_S3 & $10+5 b(R)$ & 119.1 & CT19,165 & 3.3 & 74.39 & 73.60 & 72.69 & 69.26 & 21.7 \\
\hline $\mathrm{V}(1)$ & $\mathrm{Fe} \_\mathrm{S} 3$ & $3 b$ & 0.0 & $\mathrm{C} 1,1600$ & 3.6 & 72.14 & 75.34 & 73.56 & 65.45 & 23.6 \\
\hline
\end{tabular}




\begin{tabular}{|c|c|c|c|c|c|c|c|c|c|c|}
\hline II (3) & Fe_SUEp & 7 (Pr) & 137.2 & C1iC8rt,515 & 4.8 & 21.20 & 10.24 & 14.59 & 15.53 & 29.8 \\
\hline III (4) & Fe_SUEp & $12(\mathrm{R})$ & 48.8 & CMS20,170 & 3.9 & 14.00 & 11.07 & 19.76 & 17.19 & 25.2 \\
\hline II (3) & Fe_TDFWp & 7 & 137.2 & C1iC8rt,515 & 4.0 & 12.31 & 6.97 & 9.28 & 9.62 & 25.8 \\
\hline III (4) & Fe_TDFWp & $12(\mathrm{R})$ & 44.9 & CMS20,170 & 4.2 & 8.64 & 7.28 & 11.59 & 10.84 & 26.6 \\
\hline IV (7) & Fe_TFWm & $4 b$ & 97.5 & Рy65C,506 & 3.3 & 0.64 & 0.95 & 0.93 & 0.29 & 21.4 \\
\hline
\end{tabular}

665 
667 Fig. 1 Schematic representation of the process to obtain the mapping population 668 (discarding nucellar seedlings) from the cross between Cleopatra mandarin and trifoliate 669 orange (at the top). Next step (downwards) was the obtention of nucellar seedlings from 67062 apomictic $\mathrm{R} \times \operatorname{Pr}$ hybrids, and 2 parents as controls. Two years after, these seedlings 671 (genetically identical to the mother plant) were grafted with the Clausellina satsuma 672 mandarin (C. unshiu). These grafted plants had to grow during 5 years to uniformly 673 yield fruits before establishing the Fe treatments (phenotyped population).

674 Fig. 2 Distribution of scion traits significantly affected by lowering [Fe] in nutrient 675 solution (Online Resource 2): Fruit [Fe] in ppm (Fe_F), ratio between fruit and leaf Fe 676 concentrations (Fe_F_Lf), leaf SPAD value at the end of experiment (S3), and Leaf $677[\mathrm{Ca}]$ in ppm (Ca_Lf). Opaque grey indicates trait values under control treatment, and 678 transparent colored, trait values under low-Fe treatment. The parental means are 679 indicated: full black and grey lines for Cleopatra and Flying Dragon in control, 680 respectively, while dashed black and grey lines are the corresponding in low-Fe 681 condition.

682 Fig. 3 Graphic representation of principal component analysis from the correlation 683 matrix among vegetative, mineral, and fruit yield and quality traits in the grafted Citrus $684 \mathrm{x}$ Poncirus population under control iron conditions. Most important positive 685 relationships are encircled.

686 Fig. 4 Graphic representation of principal component analysis from the correlation 687 matrix among vegetative, mineral, and fruit yield and quality traits in the grafted Citrus 688 x Poncirus population under low-Fe stress. Most important positive relationships are 689 encircled.

690 Fig. 5 Genotypic means and standard errors for significant epistatic interactions 691 between QTL markers and/or cofactors governing two traits: (A) $\mathrm{Cu}$ fruit concentration 692 under low Fe (between Fe_Cu_F QTLs markers CR17,300 and C8iC1rt,650), and (B) 693 leaf SPAD values under low Fe (between Fe_S3 QTLs markers CT19.165 and 694 C1,1600). Genotypes are coded as ac, ad, bc and bd (being C. reshni ab and P. 695 trifoliata $\mathbf{c d}$ ), at the first locus (X axis), and a square (ll, ac), a triangle (lm, ad), a circle 696 (bc) or an asterisk (bd) at the second locus. 
697 Fig. 6 LOD profiles of QTLs involved in rootstock mediated tolerance to iron 698 deficiency and fruit quality traits that group together in three genomic regions (regions 699 II to IV in Table 2). Genetic positions (markers) along the three integrated Citrus700 Poncirus linkage groups are shown under the $\mathrm{X}$ axis. Selected marker intervals, 701 estimated physical distance, and number of genes downloaded from the C. clementina 702 data base at https://phytozome.jgi.doe.gov are included.

703 


\section{Electronic Supplementary Material}

705

706

707

708

709

710

711

712

713

714

715

716

717

718

719

720

721

722

723

724

725

726

ESM_1 $P$-values of significantly $(\mathrm{p}<0.05)$ different traits between parents $($ Cleopatra and Flying Dragon) as rootstocks. Non-significant is denoted as ns.

ESM_2 $P$-values for the significant effects in the mixed model analysis.

ESM_3 Pearson coefficients of significantly correlated traits $(p \leq 0.05)$ between Fe treatments.

ESM_4 Pearson coefficients between significantly correlated traits $(p \leq 0.02)$ under control (left side) and low-Fe conditions (right side).

ESM_5 Overrepresented Biological Processes and Molecular Functions within QTL genomic regions detected for tolerance to low iron stress and related traits using the Singular Enrichment Analysis tool with the Fisher's Exact with FDR multiple test correction (Tian et al. 2017) at the AgriGo platform

\section{(http://systemsbiology.cau.edu.cn/agriGOv2/).}

ESM_6 Summary list of candidate genes (transcripts) downloaded from the $C$. clementina genome database at https://phytozome.jgi.doe.gov within the marker intervals for the QTL regions I, II, II, IV and V. The number of observed (Obs) from total (Tot) annotated genes of each type is indicated except when a different annotation was obtained from NCBI (between parenthesis). Only one transcript of each type is provided, as well its start and end physical positions in bp. 
Manuscript entitled "QTL and candidate gene analyses of rootstock-mediated mandarin fruit yield and quality traits under contrasting iron availabilities" submitted to TGG by MJ Asins (mjasins@ivia.es), MV Raga, D Roca and EA Carbonell

ESM_1 $P$-values of significantly $(\mathrm{p}<0.05)$ different traits between parents $($ Cleopatra and Flying Dragon) as rootstocks. Non-significant is denoted as ns.

\begin{tabular}{|l|c|c|}
\cline { 2 - 3 } \multicolumn{1}{l|}{ Trait } & $\mathbf{p}$ (Control) & $\mathbf{p}$ (Low-Fe) \\
\hline Ca_F & $\mathrm{ns}$ & 0.0080 \\
\hline Mg_F & 0.0070 & $\mathrm{~ns}$ \\
\hline Mg_Lf & $\mathrm{ns}$ & 0.0430 \\
\hline Mn_Lf & 0.0060 & $\mathrm{~ns}$ \\
\hline Si_Lf & 0.0100 & $\mathrm{~ns}$ \\
\hline S3 & $\mathrm{ns}$ & 0.0003 \\
\hline LA & 0.0440 & $\mathrm{~ns}$ \\
\hline dP & 0.0160 & $\mathrm{~ns}$ \\
\hline
\end{tabular}


Manuscript entitled "QTL and candidate gene analyses of rootstock-mediated mandarin fruit yield and quality traits under contrasting iron availabilities" submitted to TGG by MJ Asins (mjasins@ivia.es), MV Raga, D Roca and EA Carbonell

ESM_2 $P$-values for the significant effects in the mixed model analysis.

\begin{tabular}{|c|c|c|c|c|}
\hline Abbrev. & TRAIT & G & E & GxE \\
\hline Al_F & [Al] in fruit & & & \\
\hline $\mathrm{Ca} \_\mathrm{F}$ & [Ca] in fruit & & & \\
\hline Cu_F & {$[\mathrm{Cu}]$ in fruit } & 0.0149 & & \\
\hline $\mathrm{Fe} \_\mathrm{F}$ & {$[\mathrm{Fe}]$ in fruit } & & 0.0183 & \\
\hline K_F & {$[\mathrm{K}]$ in fruit } & 0.0087 & & \\
\hline Mg_F & {$[\mathrm{Mg}]$ in fruit } & 0.0007 & & \\
\hline Mn_F & {$[\mathrm{Mn}]$ in fruit } & & & \\
\hline $\mathrm{Na}$ & {$[\mathrm{Na}]$ in fruit } & & & \\
\hline P_F & {$[\mathrm{P}]$ in fruit } & & & \\
\hline S_F & [S] in fruit & 0.0242 & & \\
\hline Si_F & [Si] in fruit & $<0.0001$ & & \\
\hline$Z n \_F$ & [Zn] in fruit & & & \\
\hline Al_Lf & {$[\mathrm{Al}]$ in leaf } & $<0.0001$ & & \\
\hline Ca_Lf & [Ca] in leaf & 0.0077 & & 0.0048 \\
\hline Cu_Lf & {$[\mathrm{Cu}]$ in leaf } & & & \\
\hline $\mathrm{Fe} \_$Lf & {$[\mathrm{Fe}]$ in leaf } & 0.0061 & & \\
\hline K_Lf & {$[\mathrm{K}]$ in leaf } & 0.017 & & \\
\hline Mg_Lf & {$[\mathrm{Mg}]$ in leaf } & 0.0006 & & \\
\hline Mn_Lf & [Mn] in leaf & 0.0039 & & \\
\hline $\mathrm{Na}$ Lff & {$[\mathrm{Na}]$ in leaf } & 0.0085 & & \\
\hline P_Lf & {$[\mathrm{P}]$ in leaf } & 0.023 & & \\
\hline S_Lf & {$[S]$ in leaf } & 0.0296 & & \\
\hline Si_Lf & [Si] in leaf & 0.0012 & & \\
\hline Zn_Lf & [Zn] in leaf & 0.0288 & & \\
\hline Fe_F_Lf & $100(\mathrm{Fe}$ _F/Fe_Lf) & & 0.0241 & \\
\hline Al_F_Lf & 100 (Al_F/Al_Lf) & & & \\
\hline LDM & Leaf dry matter & & & \\
\hline LFW & Leaf fresh weight & & & \\
\hline LDW & Leaf dry weight & & & \\
\hline LWC & Leaf water content & & & \\
\hline S3 & SPAD at the end & 0.0021 & 0.0062 & \\
\hline LA & Leaf area & & & \\
\hline FNm & Fruit number & 0.0319 & & \\
\hline TFWm & Total fruit weight & 0.0007 & & \\
\hline TDFWp & Total pulp dry weight & 0.0031 & & \\
\hline FeUEp & Total harvested Fe & 0.0001 & & \\
\hline MgUEp & Total harvested $\mathrm{Mg}$ & 0.0051 & & \\
\hline PUEp & Total harvested $\mathrm{P}$ & 0.0037 & & \\
\hline
\end{tabular}




\begin{tabular}{|l|c|l|l|l|} 
SUEp & Total harvested Fe & $<0.0001$ & & \\
\hline FW & Fruit weight & & & \\
\hline FD & Fruit diameter & & & \\
\hline RT & Rind thickness & & & \\
\hline JV & Juice volume & & & \\
\hline JC & Juice content & & & \\
\hline SSC & Soluble-solids content & & & \\
\hline NaOH & NaOH volume & & & \\
\hline SSC_A & SSC/Acidity ratio & & & \\
\hline
\end{tabular}


Manuscript entitled "QTL and candidate gene analyses of rootstock-mediated mandarin fruit yield and quality traits under contrasting iron availabilities" submitted to TGG by MJ Asins (mjasins@ivia.es), MV Raga, D Roca and EA Carbonell

ESM_3 Pearson coefficients of significantly correlated traits $(p \leq 0.05)$ between Fe treatments.

\begin{tabular}{|c|c|c|c|}
\hline Trait 1 & Trait 2 & Pearson & $\boldsymbol{p}$-value \\
\hline Fe_Al_Lf & C_Al_Lf & 0.70 & $<0.0001$ \\
\hline Fe_FNm & C_FNm & 0.41 & 0.0008 \\
\hline Fe_K_Lf & C_K_Lf & 0.40 & 0.0011 \\
\hline Fe_Mg_Lf & C_Mg_Lf & 0.37 & 0.0033 \\
\hline Fe_Mn_Lf & C_Mn_Lf & 0.26 & 0.0392 \\
\hline Fe_P_Lf & C_P_Lf & 0.41 & 0.0008 \\
\hline Fe_PUEp & C_PUEp & 0.27 & 0.0359 \\
\hline Fe_S_Lf & C_S_Lf & 0.33 & 0.0098 \\
\hline Fe_S3 & C_S3 & 0.42 & 0.0008 \\
\hline Fe_Si_F & C_Si_F & 0.61 & $<0.0001$ \\
\hline Fe_Si_Lf & C_Si_Lf & 0.27 & 0.0343 \\
\hline Fe_SSC & C_SSC & 0.33 & 0.0103 \\
\hline Fe_TDFWp & C_TDFWp & 0.28 & 0.0298 \\
\hline Fe_TFWm & C_TFWm & 0.46 & 0.0001 \\
\hline
\end{tabular}


Manuscript entitled "QTL and candidate gene analyses of rootstock-mediated mandarin fruit yield and quality traits under contrasting iron availabilities" submitted to TGG by MJ Asins (mjasins@ivia.es), MV Raga, D Roca and EA Carbonell

ESM_4 Pearson coefficients between significantly correlated traits $(p \leq 0.02)$ under control (left side) and low-Fe conditions (right side).

\begin{tabular}{|l|l|c|r|}
\hline Trait 1 & Trait 2 & Pearson & p-value \\
\hline C_Al_F & C_Al_F_Lf & 0.74 & $<0.0001$ \\
\hline C_Al_Lf & C_Al_F_Lf & -0.50 & $<0.0001$ \\
\hline C_Al_Lf & C_Fe_F_Lf & -0.39 & 0.0015 \\
\hline C_Ca_Lf & C_Fe_F_Lf & -0.35 & 0.0055 \\
\hline C_Cu_F & C_FeUEp & 0.38 & 0.0029 \\
\hline C_Cu_F & C_Mn_Lf & 0.50 & $<0.0001$ \\
\hline C_Cu_F & C_PUEp & 0.30 & 0.0173 \\
\hline C_CU_F & C_SUEp & 0.30 & 0.0181 \\
\hline C_CU_Lf & C_Fe_F_Lf & -0.31 & 0.0157 \\
\hline C_CU_Lf & C_PUEp & 0.33 & 0.0099 \\
\hline C_Cu_Lf & C_SUEp & 0.32 & 0.0107 \\
\hline C_Fe_F & C_Ca_F & 0.47 & 0.0001 \\
\hline C_Fe_F & C_Fe_F_Lf & 0.71 & $<0.0001$ \\
\hline C_Fe_F & C_MgUEp & -0.46 & 0.0002 \\
\hline C_Fe_F & C_Na_Lf & -0.33 & 0.0094 \\
\hline C_Fe_F & C_PUEp & -0.45 & 0.0002 \\
\hline C_Fe_F & C_SUEp & -0.39 & 0.0019 \\
\hline C_Fe_F & C_TDFWp & -0.50 & $<0.0001$ \\
\hline C_Fe_F_Lf & C_MgUEp & -0.35 & 0.006 \\
\hline C_Fe_F_Lf & C_PUEp & -0.36 & 0.0049 \\
\hline C_Fe_F_Lf & C_SUEp & -0.30 & 0.0188 \\
\hline C_Fe_F_Lf & C_TDFWp & -0.38 & 0.0026 \\
\hline C_Fe_Lf & C_Ca_Lf & 0.43 & 0.0005 \\
\hline C_Fe_Lf & C_Cu_Lf & 0.42 & 0.0007 \\
\hline C_Fe_Lf & C_Fe_F_Lf & -0.31 & 0.0157 \\
\hline C_FeUEp & C_TDFWp & 0.84 & $<0.0001$ \\
\hline C_FNm & C_Cu_Lf & 0.33 & 0.0094 \\
\hline C_FNm & C_Fe_F & -0.32 & 0.0107 \\
\hline C_FNm & C_Fe_F_Lf & -0.38 & 0.0021 \\
\hline C_FNm & C_FeUEp & 0.57 & $<0.0001$ \\
\hline C_FNm & C_FW & -0.60 & $<0.0001$ \\
\hline C_FNm & C_JC & 0.36 & 0.0042 \\
\hline C_FNm & C_JV & -0.53 & $<0.0001$ \\
\hline C_FNm & C_Mg_Lf & 0.42 & 0.0008 \\
\hline C_FNm & C_MgUEp & 0.63 & $<0.0001$ \\
\hline C_FNm & C_NaOH & 0.41 & 0.0012 \\
\hline C_FNm & C_P_F & 0.40 & 0.0011 \\
\hline
\end{tabular}

\begin{tabular}{|l|l|c|r|}
\hline Trait 1 & Trait 2 & Pearson & p-value \\
\hline Fe_Al_F & Fe_Al_F_Lf & 0.81 & $<0.0001$ \\
\hline Fe_Al_Lf & Fe_AI_F_Lf & -0.47 & 0.0001 \\
\hline Fe_Ca_Lf & Fe_Fe_F_Lf & -0.46 & 0.0002 \\
\hline Fe_Ca_Lf & Fe_LDM & 0.39 & 0.0017 \\
\hline Fe_Cu_F & Fe_Fe_F_Lf & 0.39 & 0.0019 \\
\hline Fe_CU_F & Fe_FeUEp & 0.34 & 0.0067 \\
\hline Fe_CU_F & Fe_Mn_Lf & 0.30 & 0.0196 \\
\hline Fe_CU_F & Fe_PUEp & 0.31 & 0.0146 \\
\hline Fe_Cu_Lf & Fe_Al_Lf & 0.36 & 0.004 \\
\hline Fe_Fe_F & Fe_Ca_F & 0.35 & 0.0051 \\
\hline Fe_Fe_F & Fe_CU_F & 0.52 & $<0.0001$ \\
\hline Fe_Fe_F & Fe_Fe_F_Lf & 0.53 & $<0.0001$ \\
\hline Fe_Fe_F & Fe_FeUEp & 0.34 & 0.0062 \\
\hline Fe_Fe_F & Fe_Mn_Lf & 0.38 & 0.0023 \\
\hline Fe_Fe_Lf & Fe_Ca_Lf & 0.60 & $<0.0001$ \\
\hline Fe_Fe_Lf & Fe_CU_Lf & 0.37 & 0.0034 \\
\hline Fe_Fe_Lf & Fe_Fe_F_Lf & -0.67 & $<0.0001$ \\
\hline Fe_Fe_Lf & Fe_LDM & 0.34 & 0.0061 \\
\hline Fe_FeUEp & Fe_TDFWp & 0.88 & $<0.0001$ \\
\hline Fe_FNm & Fe_CU_Lf & 0.36 & 0.0046 \\
\hline Fe_FNm & Fe_FeUEp & 0.56 & $<0.0001$ \\
\hline Fe_FNm & Fe_FW & -0.51 & $<0.0001$ \\
\hline Fe_FNm & Fe_JV & -0.55 & $<0.0001$ \\
\hline Fe_FNm & Fe_Mg_Lf & 0.48 & 0.0001 \\
\hline Fe_FNm & Fe_MgUEp & 0.64 & $<0.0001$ \\
\hline Fe_FNm & Fe_Mn_Lf & 0.34 & 0.0072 \\
\hline Fe_FNm & Fe_NaOH & 0.58 & $<0.0001$ \\
\hline Fe_FNm & Fe_PUEp & 0.66 & $<0.0001$ \\
\hline Fe_FNm & Fe_Si_Lf & 0.40 & 0.0015 \\
\hline Fe_FNm & Fe_SSC & 0.70 & $<0.0001$ \\
\hline Fe_FNm & Fe_SUEp & 0.68 & $<0.0001$ \\
\hline Fe_FNm & Fe_TDFWp & 0.72 & $<0.0001$ \\
\hline Fe_FW & Fe_Ca_F & -0.33 & 0.0086 \\
\hline Fe_FW & Fe_Fe_F_Lf & 0.31 & 0.0128 \\
\hline Fe_FW & Fe_FeUEp & -0.33 & 0.0078 \\
\hline Fe_FW & Fe_JC & -0.40 & 0.0014 \\
\hline Fe_FW & Fe_JV & 0.88 & $<0.0001$ \\
\hline
\end{tabular}




\begin{tabular}{|c|c|c|c|}
\hline C_FNm & C_PUEp & 0.70 & $<0.0001$ \\
\hline C_FNm & C_Si_Lf & 0.40 & 0.0014 \\
\hline C_FNm & C_SSC & 0.54 & $<0.0001$ \\
\hline C_FNm & C_SUEp & 0.66 & $<0.0001$ \\
\hline C_FNm & C_TDFWp & 0.66 & $<0.0001$ \\
\hline C_FW & C_FeUEp & -0.39 & 0.0021 \\
\hline C_FW & C_JC & -0.59 & $<0.0001$ \\
\hline C_FW & C_JV & 0.80 & $<0.0001$ \\
\hline C_FW & C_K_Lf & 0.30 & 0.0185 \\
\hline C_FW & C_MgUEp & -0.37 & 0.0036 \\
\hline C_FW & C_NaOH & -0.60 & $<0.0001$ \\
\hline C_FW & C_PUEp & -0.43 & 0.0006 \\
\hline C_FW & C_RT & 0.63 & $<0.0001$ \\
\hline C_FW & C_SSC & -0.60 & $<0.0001$ \\
\hline C_FW & C_SSC_A & 0.47 & 0.0001 \\
\hline C_FW & C_SUEp & -0.41 & 0.0011 \\
\hline C_FW & C_TDFWp & -0.44 & 0.0003 \\
\hline C_FW & C_Zn_Lf & -0.41 & 0.0011 \\
\hline C_JC & C_Ca_F & 0.31 & 0.0144 \\
\hline C_JC & C_FeUEp & 0.32 & 0.0123 \\
\hline C_JC & C_K_F & -0.34 & 0.0076 \\
\hline C_JC & C_K_Lf & -0.33 & 0.0103 \\
\hline C_JC & C_NaOH & 0.48 & 0.0001 \\
\hline C_JC & C_SSC & 0.34 & 0.0067 \\
\hline C_JC & C_SSC_A & -0.49 & 0.0001 \\
\hline C_JV & C_Fe_F & 0.33 & 0.0085 \\
\hline C_JV & C_Fe_F_Lf & 0.40 & 0.0014 \\
\hline C_JV & C_MgUEp & -0.34 & 0.0079 \\
\hline C_JV & C_Na_F & -0.31 & 0.0139 \\
\hline C_JV & C_Na_Lf & -0.31 & 0.0161 \\
\hline C_JV & C_NaOH & -0.39 & 0.0016 \\
\hline C_JV & C_PUEp & -0.41 & 0.0009 \\
\hline C_JV & C_SSC & -0.54 & $<0.0001$ \\
\hline C_JV & C_SUEp & -0.37 & 0.0033 \\
\hline C_JV & C_TDFWp & -0.40 & 0.0014 \\
\hline C_JV & C_Zn_Lf & -0.39 & 0.0017 \\
\hline C_K_F & C_Cu_F & 0.38 & 0.0026 \\
\hline C_K_F & C_P_Lf & 0.38 & 0.0022 \\
\hline C_K_F & C_PUEp & 0.32 & 0.0128 \\
\hline C_LA & C_Cu_Lf & -0.31 & 0.0135 \\
\hline C_LA & C_K_Lf & -0.30 & 0.0198 \\
\hline C_LDW & C_LDM & 0.39 & 0.0023 \\
\hline C_LFW & C_Al_F_Lf & 0.34 & 0.007 \\
\hline C_LFW & C_LDW & 0.96 & $<0.0001$ \\
\hline C_LWC & C_LDM & -0.98 & $<0.0001$ \\
\hline C_LWC & C_LDW & -0.39 & 0.0022 \\
\hline
\end{tabular}

\begin{tabular}{|c|c|c|c|}
\hline $\mathrm{Fe} \_\mathrm{FW}$ & Fe_LDM & -0.30 & 0.0176 \\
\hline $\mathrm{Fe} \_\mathrm{FW}$ & Fe_MgUEp & -0.40 & 0.0011 \\
\hline Fe_FW & $\mathrm{Fe} \_\mathrm{NaOH}$ & -0.60 & $<0.0001$ \\
\hline Fe_FW & e_P_F & -0.37 & 0.0027 \\
\hline Fe_FW & Fe_PUEp & -0.49 & $<0.0001$ \\
\hline Fe_FW & Fe_RT & 0.47 & 0.0001 \\
\hline $\mathrm{Fe} \_\mathrm{FW}$ & Fe_SSC & -0.45 & 0.0002 \\
\hline Fe_FW & Fe_SSC_A & 0.42 & 0.0006 \\
\hline $\mathrm{Fe} \_\mathrm{FW}$ & Fe_SUEp & -0.46 & 0.0002 \\
\hline Fe_FW & Fe_TDFWp & -0.43 & 0.0006 \\
\hline Fe_JC & Fe_Ca_F & 0.39 & 0.0018 \\
\hline Fe_JV & $\mathrm{Fe} \_\mathrm{Fe}$ & -0.37 & 0.0033 \\
\hline Fe_JV & Fe_Mg_Lf & -0.34 & 0.0069 \\
\hline $\mathrm{Fe} \_J V$ & Fe_MgUEp & -0.50 & $<0.0001$ \\
\hline $\mathrm{Fe} \_\mathrm{JV}$ & $\mathrm{Fe} \_\mathrm{NaOH}$ & -0.61 & $<0.0001$ \\
\hline $\mathrm{Fe} \_\mathrm{JV}$ & Fe_P_F & -0.44 & 0.0004 \\
\hline Fe_JV & Fe_PUEp & -0.59 & $<0.0001$ \\
\hline Fe_JV & Fe_SSC & -0.52 & $<0.0001$ \\
\hline Fe_JV & Fe_SSC_A & 0.37 & 0.003 \\
\hline Fe_JV & Fe_S & -0.53 & $<0.0001$ \\
\hline Fe_JV & $\mathrm{Fe}$ & -0.51 & $<0$. \\
\hline Fe_K_F & Fe_Cu_F & 0.39 & 0.0019 \\
\hline Fe_K_F & $\mathrm{Fe} \_\mathrm{I}$ & 0.32 & 0.0124 \\
\hline Fe_K_F & Fe_PUEp & 0.39 & 0.002 \\
\hline Fe_LDM & Fe_Fe_F_Lf & -0.32 & 0.0113 \\
\hline Fe_LDW & Fe_LDM & 0.59 & $<0.0001$ \\
\hline $\mathrm{Fe}$ _LFW & Fe_LDM & 0.33 & 0.0091 \\
\hline $\mathrm{Fe}$ _LFW & $\mathrm{Fe} \_\mathrm{L}$ & 0.95 & $<0.0001$ \\
\hline $\mathrm{Fe}$ _LFW & Fe_LWC & -0.35 & 0.0047 \\
\hline Fe_LWC & Fe_Ca_Lf & -0.39 & 0.0017 \\
\hline Fe_LWC & Fe_Fe_F_Lf & 0.32 & 0.0109 \\
\hline Fe_LWC & $\mathrm{Fe} \_\mathrm{Fe} \_\mathrm{Lf}$ & -0.34 & 0.0067 \\
\hline Fe_LWC & $\mathrm{Fe} \_\mathrm{F}$ & -0.30 & 0.0187 \\
\hline Fe_LWC & Fe_FW & 0.32 & 0.0125 \\
\hline Fe_LWC & Fe_LDM & -0.99 & $<0.0001$ \\
\hline Fe_LWC & Fe_LDW & -0.61 & $<0.0001$ \\
\hline Fe_LWC & Fe_Mg_Lf & -0.33 & 0.008 \\
\hline Fe_LWC & Fe_S_Lf & -0.33 & 0.0083 \\
\hline Fe_Mg_Lf & Fe_Ca_Lf & 0.39 & 0.0018 \\
\hline Fe_Mg_Lf & Fe_Fe_F_Lf & -0.42 & 0.0007 \\
\hline Fe_Mg_Lf & Fe_LDM & 0.31 & 0.0157 \\
\hline Fe_Mg_Lf & Fe_MgUEp & 0.33 & 0.0094 \\
\hline Fe_Mg_Lf & Fe_PUEp & 0.38 & 0.0024 \\
\hline Fe_Mg_Lf & Fe_SUEp & 0.34 & 0.0075 \\
\hline Fe_MgUEp & Fe_FeUEp & 0.89 & $<0.0001$ \\
\hline Fe_MgUEp & Fe_TDFWp & 0.93 & $<0.0001$ \\
\hline
\end{tabular}




\begin{tabular}{|c|c|c|c|}
\hline C_LWC & C_Na_F & 0.36 & 0.0041 \\
\hline C_Mg_Lf & C_Ca_Lf & 0.48 & 0.0001 \\
\hline C_Mg_Lf & Fe_F_Lf & -0.40 & 0.0014 \\
\hline C_Mg_Lf & C_MgUEp & 0.34 & 0.008 \\
\hline C_Mg_Lf & C_PUEp & 0.31 & 0.0151 \\
\hline C_MgUEp & C_FeUEp & 0.81 & $<0.0001$ \\
\hline C_MgUEp & C_TDFWp & 0.96 & $<0.0001$ \\
\hline C_Mn_F & C_Ca_F & 0.41 & 0.001 \\
\hline C_Mn_F & C_Cu_F & 0.31 & 0.015 \\
\hline C_Mn_F & C_Fe_F & 0.63 & $<0.0001$ \\
\hline C_Mn_F & C_Fe_F_Lf & 0.39 & 0.0017 \\
\hline C_Mn_F & C_Mn_Lf & 0.38 & 0.002 \\
\hline C_Mn_Lf & C_Fe_Lf & 0.48 & 0.0001 \\
\hline C_Na_F & C_Ca_Lf & 0.50 & $<0.0001$ \\
\hline C_Na_F & C_LDM & -0.31 & 0.0136 \\
\hline C_Na_F & C_Mg_Lf & 0.33 & 0.008 \\
\hline C_Na_F & C_Na_Lf & 0.43 & 0.0004 \\
\hline C_Na_F & C_PUEp & 0.31 & 0.0136 \\
\hline C_Na_F & C_Si_Lf & 0.36 & 0.0043 \\
\hline C_Na_F & C_Zn_Lf & 0.35 & 0.0047 \\
\hline C_Na_Lf & & 44 & 0.0004 \\
\hline C_Na_Lf & C_MgUEp & 0.35 & 0.0064 \\
\hline C_Na_Lf & C_PUEp & 38 & 0.0024 \\
\hline C_Na_Lf & C_SUEp & 0.36 & 0.0046 \\
\hline C_Na_Lf & C_TDFWp & 0.32 & 0.0125 \\
\hline C_NaOH & C_MgUEp & 0.30 & 0.0191 \\
\hline C_NaOH & C_PUEp & 0.35 & 0.0052 \\
\hline C_NaOH & C_S_Lf & -0.33 & 0.0099 \\
\hline C_NaOH & C_SSC_A & -0.83 & $<0.0001$ \\
\hline C_NaOH & C_SUEp & 0.32 & 0.0108 \\
\hline C_NaOH & C_TDFWp & 0.33 & 0.0098 \\
\hline C_P_F & C_Ca_Lf & 0.30 & 0.0172 \\
\hline C_P_F & C_Cu_F & 0.55 & $<0.0001$ \\
\hline C_P_F & C_K_F & 0.73 & $<0.0001$ \\
\hline C_P_F & C_Mg_Lf & 0.36 & 0.0037 \\
\hline C_P_F & C_MgUEp & 0.37 & 0.0033 \\
\hline C_P_F & C_Na_F & 0.42 & 0.0006 \\
\hline C_P_F & C_P_Lf & 0.41 & 0.0008 \\
\hline C_P_F & C_PUEp & 0.50 & $<0.0001$ \\
\hline C_P_F & C_Si_Lf & 0.39 & 0.002 \\
\hline C_P_F & C_SUEp & 0.45 & 0.0003 \\
\hline C_P_Lf & C_K_Lf & 0.57 & $<0.0001$ \\
\hline C_P_Lf & C_Mg_Lf & 0.52 & $<0.0001$ \\
\hline C_P_Lf & C_Mn_Lf & 0.35 & 0.0052 \\
\hline C_PUEp & C_FeUEp & 0.81 & $<0.0001$ \\
\hline C_PUEp & C_MgUEp & 0.96 & $<0.0001$ \\
\hline
\end{tabular}

\begin{tabular}{|c|c|c|c|}
\hline $\mathrm{Fe} \_\mathrm{Mn} \_\mathrm{F}$ & Fe_Ca_F & 0.40 & 0.0013 \\
\hline Fe_Mn_F & e_Cu_F & 0.51 & $<0.0001$ \\
\hline $\mathrm{Fe} \_\mathrm{Mn} \_\mathrm{F}$ & e_Fe_F & 0.53 & $<0.0001$ \\
\hline $\mathrm{Fe} \_\mathrm{Mn} \_\mathrm{F}$ & e_Fe_F_Lf & 0.45 & 0.0002 \\
\hline $\mathrm{Fe} \_\mathrm{Mn} \_\mathrm{F}$ & e_FeUEp & 0.37 & 0.0033 \\
\hline $\mathrm{Fe} \_\mathrm{Mn} \_\mathrm{F}$ & e_Mn_Lf & 0.63 & $<0.0001$ \\
\hline $\mathrm{Fe} \_\mathrm{Mn} \_\mathrm{F}$ & Fe_Na_Lf & -0.36 & 0.0046 \\
\hline Fe_Mn_Lf & Fe_FeUEp & 0.30 & 0.0192 \\
\hline Fe_Mn_Lf & Fe_K_Lf & 0.43 & 0.0006 \\
\hline $\mathrm{Fe} \_\mathrm{Na} \_\mathrm{F}$ & Fe_Mn_F & -0.30 & 0.0176 \\
\hline $\mathrm{Fe} \_\mathrm{Na} \_\mathrm{F}$ & $\mathrm{Fe} \_\mathrm{Na} \_\mathrm{Lf}$ & 0.50 & $<0.0001$ \\
\hline $\mathrm{Fe} \_\mathrm{Na} \_L f$ & Fe_Ca_Lf & 0.35 & 0.0052 \\
\hline $\mathrm{Fe} \_\mathrm{Na} \_L f$ & Fe_Mg_Lf & 0.32 & 0.0105 \\
\hline $\mathrm{Fe} \_\mathrm{Na} \_\mathrm{Lf}$ & Fe_Mn_Lf & -0.30 & 0.0183 \\
\hline $\mathrm{Fe} \_\mathrm{NaOH}$ & Fe_Fe_F_Lf & -0.31 & 0.0135 \\
\hline $\mathrm{Fe} \_\mathrm{NaOH}$ & Fe_FeUEp & 0.35 & 0.005 \\
\hline $\mathrm{Fe} \_\mathrm{NaOH}$ & e_K_F & 0.31 & 0.0139 \\
\hline $\mathrm{Fe} \_\mathrm{NaOH}$ & Fe_Mg_Lf & 0.38 & 0.002 \\
\hline $\mathrm{Fe} \_\mathrm{NaOH}$ & Fe_MgUEp & 0.48 & 0.0001 \\
\hline $\mathrm{Fe} \_\mathrm{NaOH}$ & Fe_P_F & 0.42 & 0.0008 \\
\hline $\mathrm{Fe} \_\mathrm{NaOH}$ & E_PUEp & 0.59 & \\
\hline $\mathrm{Fe} \_\mathrm{NaOH}$ & Fe_S_F & 0.35 & 0.0047 \\
\hline $\mathrm{Fe} \_\mathrm{Na}$ & LLf & 0.40 & 013 \\
\hline $\mathrm{Fe} \_\mathrm{Na}$ & Fe_SSC_A & -0.78 & $<0.0001$ \\
\hline $\mathrm{Fe} \_\mathrm{NaOH}$ & $\mathrm{Fe} \_$S & 0.57 & $<0.0001$ \\
\hline $\mathrm{Fe} \_\mathrm{NaOH}$ & $\mathrm{Fe}_{-}$ & 0.48 & 0.0001 \\
\hline Fe_P_F & $\mathrm{Fe} \_\mathrm{Cu}$ _F & 0.53 & $<0.0001$ \\
\hline $\mathrm{Fe} \_\mathrm{P} \_\mathrm{F}$ & $\mathrm{Fe} \_\mathrm{K} \_\mathrm{F}$ & 0.80 & $<0.0001$ \\
\hline Fe_P_F & Fe_Mg_Lf & 0.39 & 0.002 \\
\hline Fe_P_F & Fe_MgUEp & 0.41 & .001 \\
\hline Fe_P_F & Fe_PUEp & 0.55 & $<0.0001$ \\
\hline $\mathrm{Fe} \_\mathrm{P} \_\mathrm{F}$ & Fe_Si_Lf & 0.30 & 0.0186 \\
\hline $\mathrm{Fe} \_\mathrm{P} \_\mathrm{F}$ & e_SUEp & 0.46 & 0.0002 \\
\hline $\mathrm{Fe}_{-} \mathrm{P} \_\mathrm{Lf}$ & Fe_K_Lf & 0.74 & $<0.0001$ \\
\hline $\mathrm{Fe}_{-} \mathrm{P} \_\mathrm{Lf}$ & Fe_Mg_Lf & 0.52 & $<0.0001$ \\
\hline $\mathrm{Fe}$ _P_Lf & Fe_Mn_Lf & 0.45 & 0.0003 \\
\hline Fe_PUEp & Fe_FeUEp & 0.83 & $<0.0001$ \\
\hline Fe_PUEp & Fe_MgUEp & 0.96 & $<0.0001$ \\
\hline Fe_PUEp & Fe_TDFWp & 0.90 & $<0.0001$ \\
\hline $\mathrm{Fe} \_\mathrm{RT}$ & Fe_JC & -0.65 & $<0.0001$ \\
\hline Fe_RT & Fe_K_Lf & 0.32 & 0.0108 \\
\hline Fe_RT & Fe_P_Lf & 0.34 & 0.0076 \\
\hline $\mathrm{Fe} \_\mathrm{S} \_\mathrm{F}$ & Fe_Ca_F & 0.46 & 0.0002 \\
\hline $\mathrm{Fe} \_\mathrm{S} \_\mathrm{F}$ & Fe_Cu_F & 0.53 & $<0.0001$ \\
\hline Fe_S_F & Fe_FeUEp & 0.35 & 0.0047 \\
\hline Fe_S_F & Fe_K_F & 0.59 & $<0.0001$ \\
\hline
\end{tabular}




\begin{tabular}{|c|c|c|c|}
\hline C_PUEp & C_TDFWp & 0.95 & $<0.0001$ \\
\hline C_RT & C_JC & -0.72 & $<0.0001$ \\
\hline C_RT & C_NaOH & -0.43 & 0.0006 \\
\hline C_RT & C_SSC_A & 0.56 & $<0.0001$ \\
\hline C_S_F & C_Cu_F & 0.50 & $<0.0001$ \\
\hline C_S_F & C_Fe_F & 0.32 & 0.0106 \\
\hline C_S_F & C_K_F & 0.59 & $<0.0001$ \\
\hline C_S_F & C_P_F & 0.71 & $<0.0001$ \\
\hline C_S_Lf & C_Ca_Lf & 0.46 & 0.0001 \\
\hline C_S_Lf & C_Fe_Lf & 0.50 & $<0.0001$ \\
\hline C_S_Lf & C_K_Lf & 0.44 & 0.0003 \\
\hline C_S_Lf & C_Mn_Lf & 0.40 & 0.0012 \\
\hline C_S_Lf & C_P_Lf & 0.34 & 0.0066 \\
\hline C_S_Lf & C_TDFWp & -0.31 & 0.0156 \\
\hline C_S3 & C_Cu_F & 0.34 & 0.0066 \\
\hline C_S3 & C_Mg_Lf & -0.32 & 0.0113 \\
\hline C_Si_F & C_Al_F & 0.39 & 0.002 \\
\hline C_Si_F & C_Al_Lf & 0.45 & 0.0003 \\
\hline C_Si_F & C_K_F & 0.40 & 0.0014 \\
\hline C_Si_F & C_S_F & 0.30 & 0.0179 \\
\hline C_Si_F & C_Zn_Lf & 0.33 & 0.0078 \\
\hline C_Si_Lf & C_Ca_Lf & 0.47 & 0.0001 \\
\hline C_Si_Lf & C_Cu_Lf & 0.33 & 0.0094 \\
\hline C_Si_Lf & C_Fe_F_Lf & -0.47 & 0.0001 \\
\hline C_Si_Lf & C_Fe_Lf & 0.44 & 0.0004 \\
\hline C_Si_Lf & C_Mg_Lf & 0.57 & $<0.0001$ \\
\hline C_Si_Lf & C_Mn_Lf & 0.35 & 0.0055 \\
\hline C_Si_Lf & C_P_Lf & 0.53 & $<0.0001$ \\
\hline C_SSC & C_Fe_F_Lf & -0.39 & 0.0018 \\
\hline C_SSC & C_FeUEp & 0.42 & 0.0008 \\
\hline C_SSC & C_K_Lf & -0.30 & 0.019 \\
\hline C_SSC & C_MgUEp & 0.47 & 0.0001 \\
\hline C_SSC & C_NaOH & 0.62 & $<0.0001$ \\
\hline C_SSC & C_PUEp & 0.49 & 0.0001 \\
\hline C_SSC & C_S_Lf & -0.32 & 0.0119 \\
\hline C_SSC & C_SUEp & 0.48 & 0.0001 \\
\hline C_SSC & C_TDFWp & 0.52 & $<0.0001$ \\
\hline C_SUEp & C_FeUEp & 0.84 & $<0.0001$ \\
\hline C_SUEp & C_MgUEp & 0.96 & $<0.0001$ \\
\hline C_SUEp & C_PUEp & 0.98 & $<0.0001$ \\
\hline C_SUEp & C_TDFWp & 0.94 & $<0.0001$ \\
\hline C_TFWm & C_FeUEp & 0.60 & $<0.0001$ \\
\hline C_TFWm & C_FNm & 0.85 & $<0.0001$ \\
\hline C_TFWm & C_FW & -0.32 & 0.011 \\
\hline C_TFWm & C_Mg_Lf & 0.33 & 0.0085 \\
\hline C_TFWm & C_MgUEp & 0.59 & $<0.0001$ \\
\hline
\end{tabular}

\begin{tabular}{|c|c|c|c|}
\hline $\mathrm{Fe} \_\mathrm{S} \_\mathrm{F}$ & Fe_MgUEp & 0.39 & 0.0019 \\
\hline Fe_S_F & Fe_P_F & 0.80 & $<0.0001$ \\
\hline Fe_S_F & Fe_PUEp & 0.51 & $<0.0001$ \\
\hline Fe_S_F & Fe_SUEp & 0.56 & $<0.0001$ \\
\hline Fe_S_Lf & Fe_Ca_Lf & 0.52 & $<0.0001$ \\
\hline Fe_S_Lf & Fe_Fe_F_Lf & -0.46 & 0.0002 \\
\hline Fe_S_Lf & Fe_Fe_Lf & 0.59 & $<0.0001$ \\
\hline Fe_S_Lf & Fe_K_Lf & 0.42 & 0.0007 \\
\hline Fe_S_Lf & Fe_LDM & 0.35 & 0.0059 \\
\hline Fe_S_Lf & Fe_Mn_Lf & 0.43 & 0.0005 \\
\hline Fe_S_Lf & Fe_P_Lf & 0.35 & 0.0053 \\
\hline Fe_S3 & Fe_Fe_F & 0.40 & 0.0014 \\
\hline Fe_S3 & Fe_JV & -0.30 & 0.0169 \\
\hline $\mathrm{Fe} \_\mathrm{Si}$ _F & Fe_Al_Lf & 0.61 & $<0.0001$ \\
\hline $\mathrm{Fe} \_\mathrm{Si}$-F & $\mathrm{Fe}$ _Ca_F & 0.41 & 0.0008 \\
\hline $\mathrm{Fe} \_\mathrm{Si} \_\mathrm{F}$ & $\mathrm{Fe} \_\mathrm{K} \_\mathrm{F}$ & 0.32 & 0.0109 \\
\hline $\mathrm{Fe} \_\mathrm{Si}$ F & $\mathrm{Fe} \_\mathrm{Na}$ _F & -0.35 & 0.0052 \\
\hline $\mathrm{Fe} \_\mathrm{Si}$ _F & Fe_P_F & 0.37 & 0.003 \\
\hline $\mathrm{Fe} \_\mathrm{Si}$ F & Fe_S_F & 0.46 & 0.0002 \\
\hline $\mathrm{Fe} \_\mathrm{Si}$ F & Fe_Zn_LF & 0.31 & 0.0146 \\
\hline Fe_Si_Lf & Fe_Ca_Lf & 0.52 & $<0.0001$ \\
\hline Fe_Si_Lf & Fe_Cu_Lf & 0.45 & 0.0003 \\
\hline Fe_Si_Lf & Fe_Fe_F_Lf & -0.56 & $<0.0001$ \\
\hline Fe_Si_Lf & Fe_Fe_Lf & 0.53 & $<0.0001$ \\
\hline Fe_Si_Lf & Fe_Mg_Lf & 0.64 & $<0.0001$ \\
\hline Fe_Si_Lf & $\mathrm{Fe} \_\mathrm{Na}$ _Lf & 0.48 & 0.0001 \\
\hline Fe_Si_Lf & Fe_P_Lf & 0.34 & 0.0071 \\
\hline Fe_Si_Lf & Fe_PUEp & 0.32 & 0.0111 \\
\hline Fe_Si_Lf & Fe_SUEp & 0.30 & 0.0169 \\
\hline Fe_SSC & Fe_Cu_Lf & 0.30 & 0.0176 \\
\hline Fe_SSC & Fe_Fe_F_Lf & -0.30 & 0.0173 \\
\hline $\mathrm{Fe} \_\mathrm{SSC}$ & Fe_K_Lf & 0.30 & 0.0165 \\
\hline $\mathrm{Fe} \_\mathrm{SSC}$ & Fe_Mg_Lf & 0.37 & 0.0032 \\
\hline $\mathrm{Fe} \_\mathrm{SSC}$ & Fe_MgUEp & 0.38 & 0.0025 \\
\hline $\mathrm{Fe} \_\mathrm{SSC}$ & $\mathrm{Fe} \_\mathrm{NaOH}$ & 0.72 & $<0.0001$ \\
\hline Fe_SSC & Fe_P_Lf & 0.32 & 0.012 \\
\hline Fe_SSC & Fe_PUEp & 0.48 & 0.0001 \\
\hline Fe_SSC & Fe_Si_Lf & 0.41 & 0.0009 \\
\hline Fe_SSC & $\mathrm{Fe} \_\mathrm{S}$ & 0.51 & $<0.0001$ \\
\hline Fe_SSC & Fe_TDFWp & 0.55 & $<0.0001$ \\
\hline Fe_SSC_A & Fe_K_F & -0.45 & 0.0002 \\
\hline Fe_SSC_A & Fe_MgUEp & -0.31 & 0.0153 \\
\hline Fe_SSC_A & Fe_P_F & -0.49 & $<0.0001$ \\
\hline Fe_SSC_A & Fe_PUEp & -0.36 & 0.004 \\
\hline Fe_SSC_A & Fe_S_F & -0.39 & 0.0018 \\
\hline Fe_SSC_A & Fe_SUEp & -0.32 & 0.0123 \\
\hline
\end{tabular}




\begin{tabular}{|l|l|l|r|} 
C_TFWm & C_Mn_Lf & 0.31 & 0.014 \\
\hline C_TFWm & C_PUEp & 0.62 & $<0.0001$ \\
\hline C_TFWm & C_Si_Lf & 0.31 & 0.0143 \\
\hline C_TFWm & C_SSC & 0.36 & 0.0042 \\
\hline C_TFWm & C_SUEp & 0.59 & $<0.0001$ \\
\hline C_TFWm & C_TDFWp & 0.62 & $<0.0001$ \\
\hline C_Zn_F & C_Al_F & 0.39 & 0.0018 \\
\hline C_Zn_F & C_Na_F & 0.31 & 0.0158 \\
\hline C_Zn_F & C_P_F & 0.39 & 0.0016 \\
\hline C_Zn_F & C_S_F & 0.35 & 0.0052 \\
\hline C_Zn_F & C_Si_F & 0.30 & 0.0166 \\
\hline C_Zn_Lf & C_Al_Lf & 0.40 & 0.0013 \\
\hline C_Zn_Lf & C_Ca_Lf & 0.37 & 0.0032 \\
\hline
\end{tabular}

\begin{tabular}{|l|l|c|r|} 
Fe_SUEp & Fe_FeUEp & 0.87 & $<0.0001$ \\
\hline Fe_SUEp & Fe_MgUEp & 0.94 & $<0.0001$ \\
\hline Fe_SUEp & Fe_PUEp & 0.97 & $<0.0001$ \\
\hline Fe_SUEp & Fe_TDFWp & 0.91 & $<0.0001$ \\
\hline Fe_TFWm & Fe_FeUEp & 0.44 & 0.0004 \\
\hline Fe_TFWm & Fe_FNm & 0.84 & $<0.0001$ \\
\hline Fe_TFWm & Fe_K_Lf & 0.34 & 0.0071 \\
\hline Fe_TFWm & Fe_Mg_Lf & 0.41 & 0.0008 \\
\hline Fe_TFWm & Fe_MgUEp & 0.47 & 0.0001 \\
\hline Fe_TFWm & Fe_Mn_Lf & 0.43 & 0.0004 \\
\hline Fe_TFWm & Fe_P_Lf & 0.33 & 0.0085 \\
\hline Fe_TFWm & Fe_PUEp & 0.47 & 0.0001 \\
\hline Fe_TFWm & Fe_SSC & 0.55 & $<0.0001$ \\
\hline Fe_TFWm & Fe_SUEp & 0.50 & $<0.0001$ \\
\hline Fe_TFWm & Fe_TDFWp & 0.57 & $<0.0001$ \\
\hline Fe_Zn_F & Fe_Al_F_Lf & 0.32 & 0.0102 \\
\hline Fe_Zn_F & Fe_Ca_Lf & -0.30 & 0.0167 \\
\hline Fe_Zn_F & Fe_Si_Lf & -0.30 & 0.018 \\
\hline Fe_Zn_LF & Fe_Al_Lf & 0.38 & 0.0023 \\
\hline Fe_Zn_LF & Fe_Ca_Lf & 0.46 & 0.0001 \\
\hline Fe_Zn_LF & Fe_Fe_F_Lf & -0.36 & 0.0044 \\
\hline Fe_Zn_LF & Fe_Fe_Lf & 0.36 & 0.0045 \\
\hline Fe_Zn_LF & Fe_Si_Lf & 0.36 & 0.0044 \\
\hline
\end{tabular}


ESM_5 Overrepresented Biological Processes and Molecular Functions within QTL genomic regions.

\section{Biological Process in Region II}

\section{$-\log F D R$}

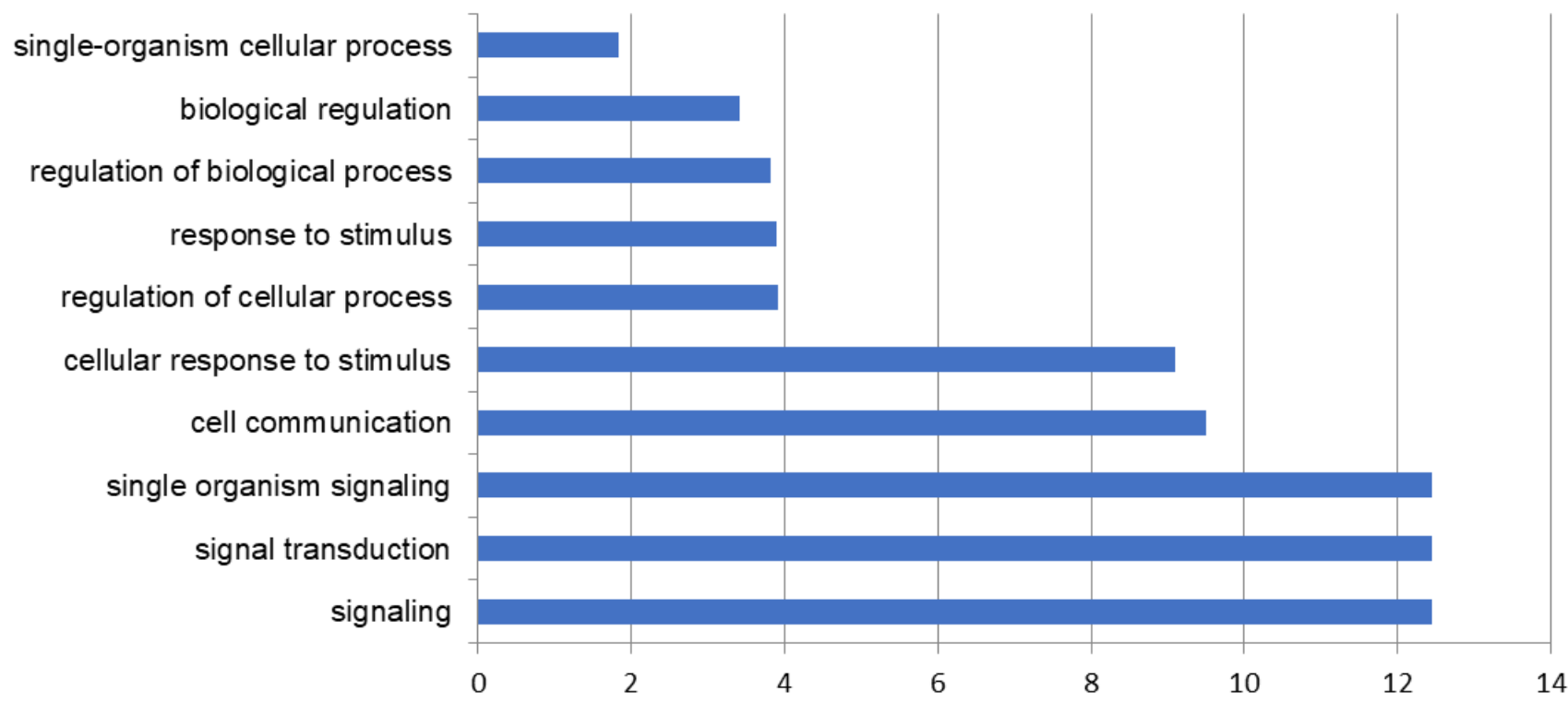

\section{Molecular Function in Region II}

\section{$-\log F D R$}

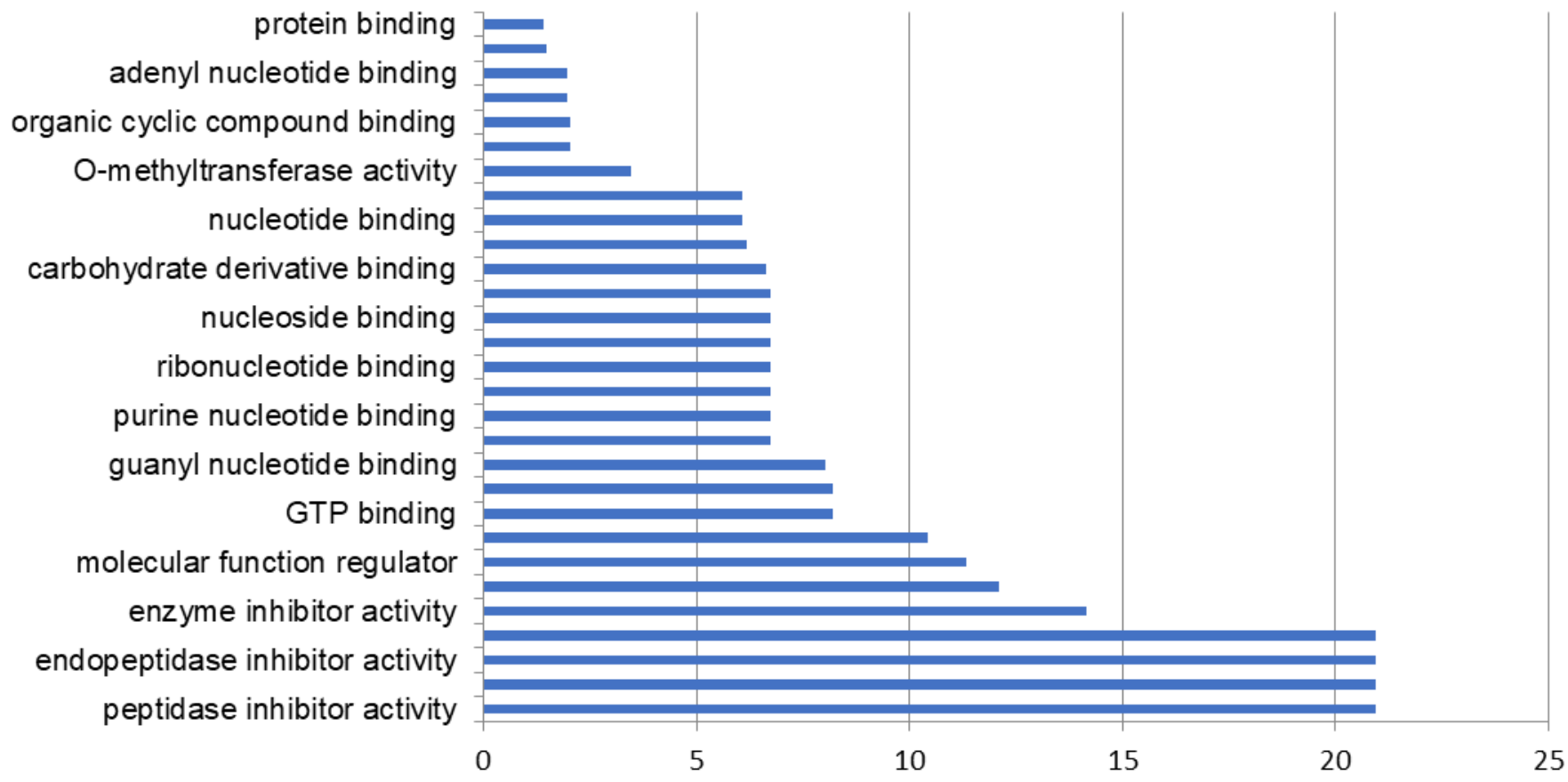




\section{$-\log F D R$}

primary metabolic process organic substance metabolic process protein phosphorylation phosphorus metabolic process phosphate-containing compound metabolic process macromolecule metabolic process protein modification process macromolecule modification cellular protein modification process phosphorylation proteolysis protein metabolic process

\section{Molecular Function in Region III - -logFDR}

transferase activity, transferring phosphorus-containing groups

$$
\text { kinase activity }
$$

protein kinase activity

phosphotransferase activity, alcohol group as acceptor

serine hydrolase activity serine-type peptidase activity

peptidase activity, acting on L-amino acid peptides peptidase activity

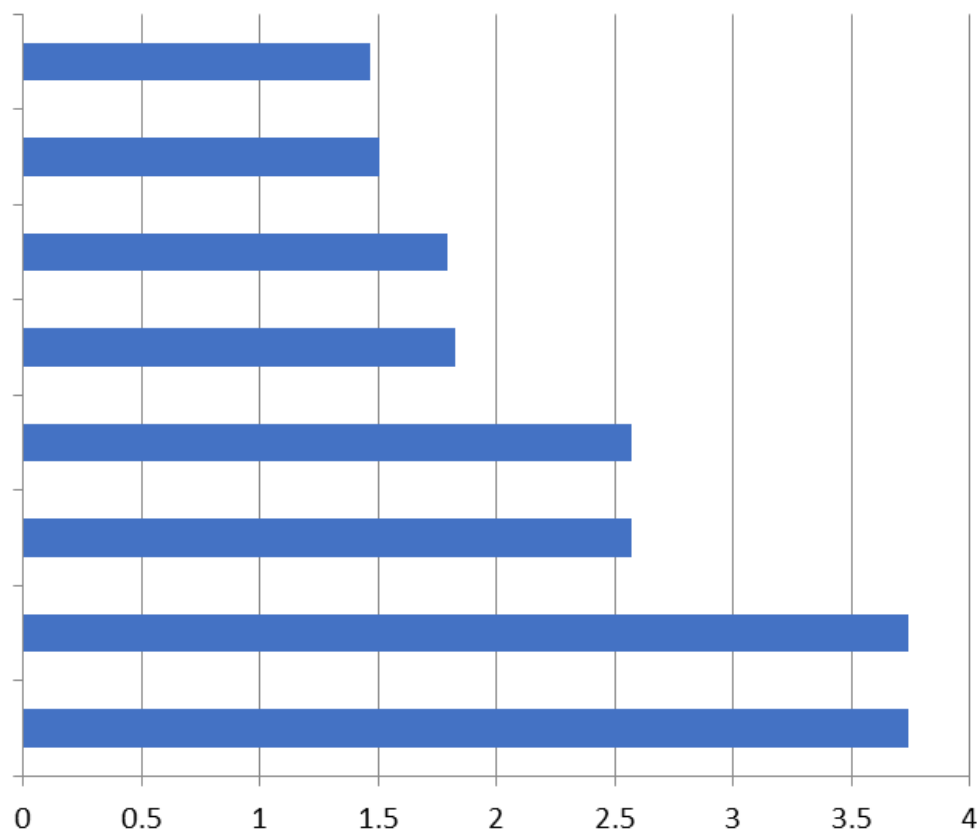




\title{
Biological Process in Region IV $\quad$-logFDR
}

\author{
DNA metabolic process \\ cellular response to stress
}

cellular response to DNA damage stimulus

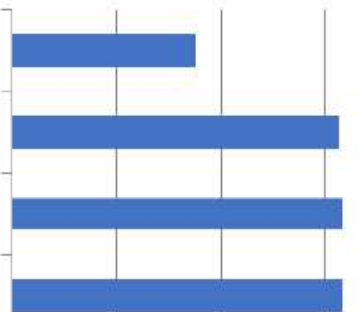

DNA repair

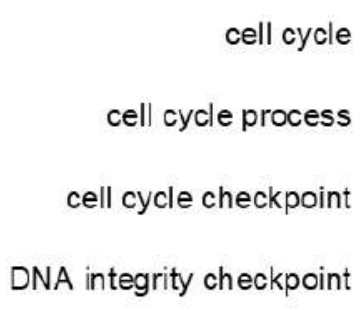

DNA damage checkpoint
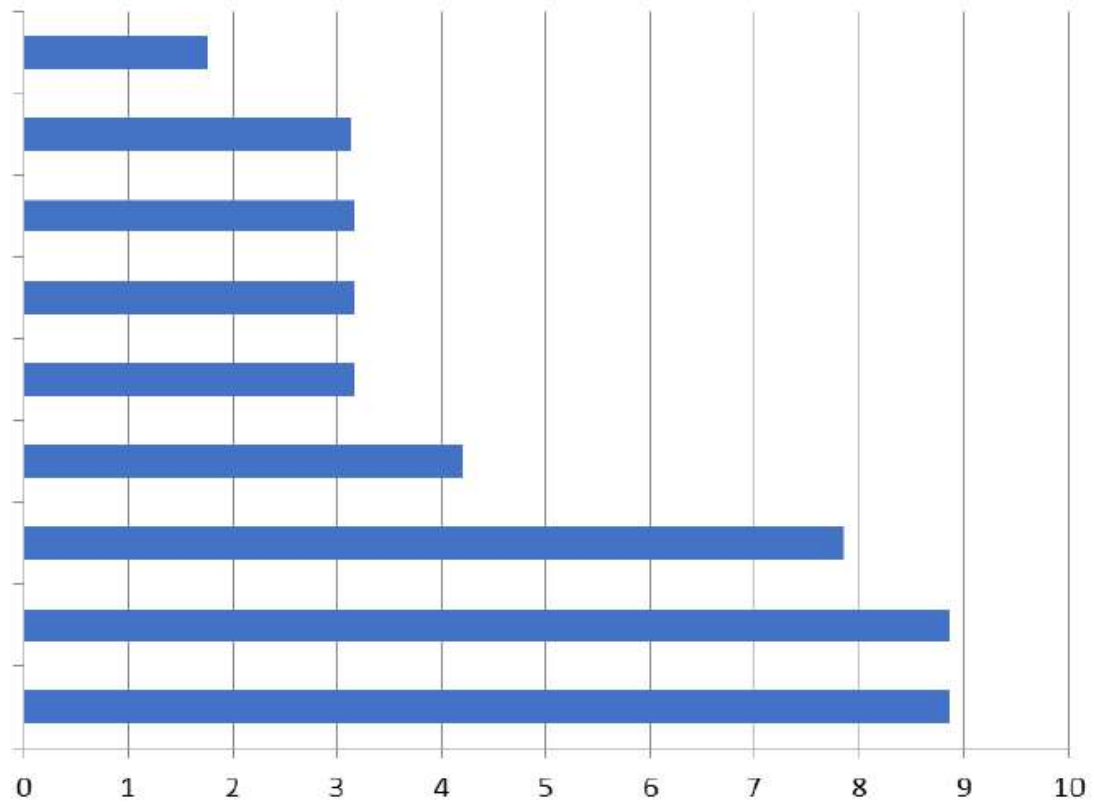

\section{Molecular Function in Region IV $\quad$-logFDR}

nucleoside-triphosphatase activity polysaccharide binding pattern binding

hydrolase activity, acting on acid anhydrides

hydrolase activity, acting on acid anhydrides, in phosphorus-containing pyrophosphatase activity ATPase activity binding protein binding organic cyclic compound binding heterocyclic compound binding small molecule binding nucleoside phosphate binding nucleotide binding carbohydrate derivative binding purine ribonucleoside binding nucleoside binding purine nucleoside binding ribonucleotide binding purine ribonucleotide binding purine nucleotide binding ribonucleoside binding adenyl nucleotide binding adenyl ribonucleotide binding ADP binding

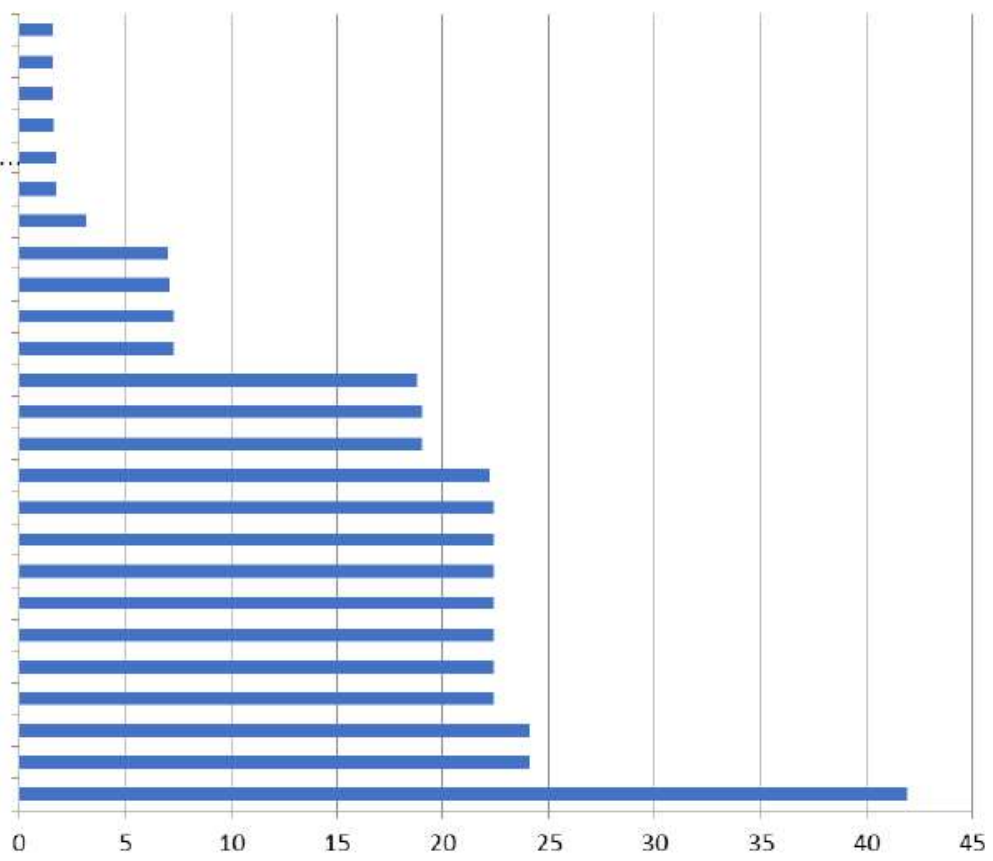


Biological Process in Region V

\section{-LogFDR}

metabolic process

organic substance metabolic process

primary metabolic process

cellular metabolic process

single-organism process

macromolecule metabolic process

cellular process

cellular macromolecule metabolic process

protein metabolic process

cellular protein metabolic process

single-organism cellular process

macromolecule modification

protein modification process

cellular protein modification process

phosphorus metabolic process

phosphate-containing compound metabolic process

protein phosphorylation

phosphorylation

cell communication

multicellular organismal process

single organism reproductive process

reproductive process

reproduction

multi-organism process

recognition of pollen

pollination

pollen-pistil interaction

multi-multicellular organism process

multi-organism reproductive process

cell recognition
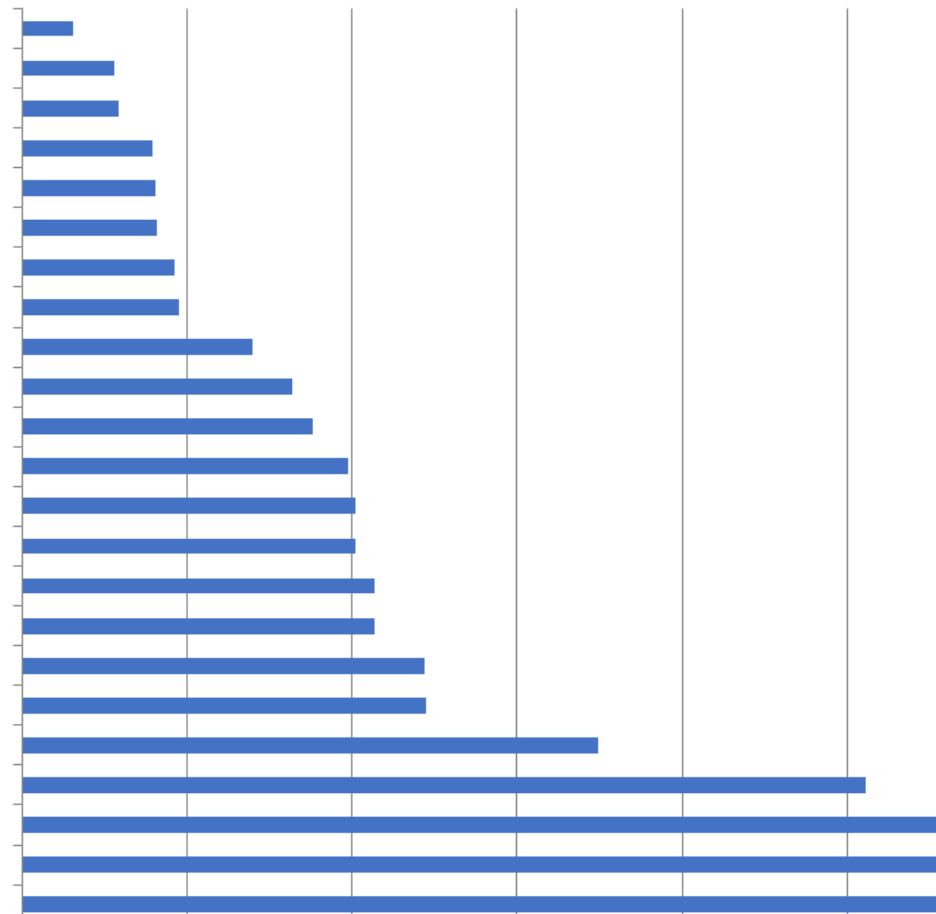

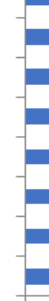

0

10

20

30

40

\section{Molecular Function in Region V}

\section{-LogFDR}

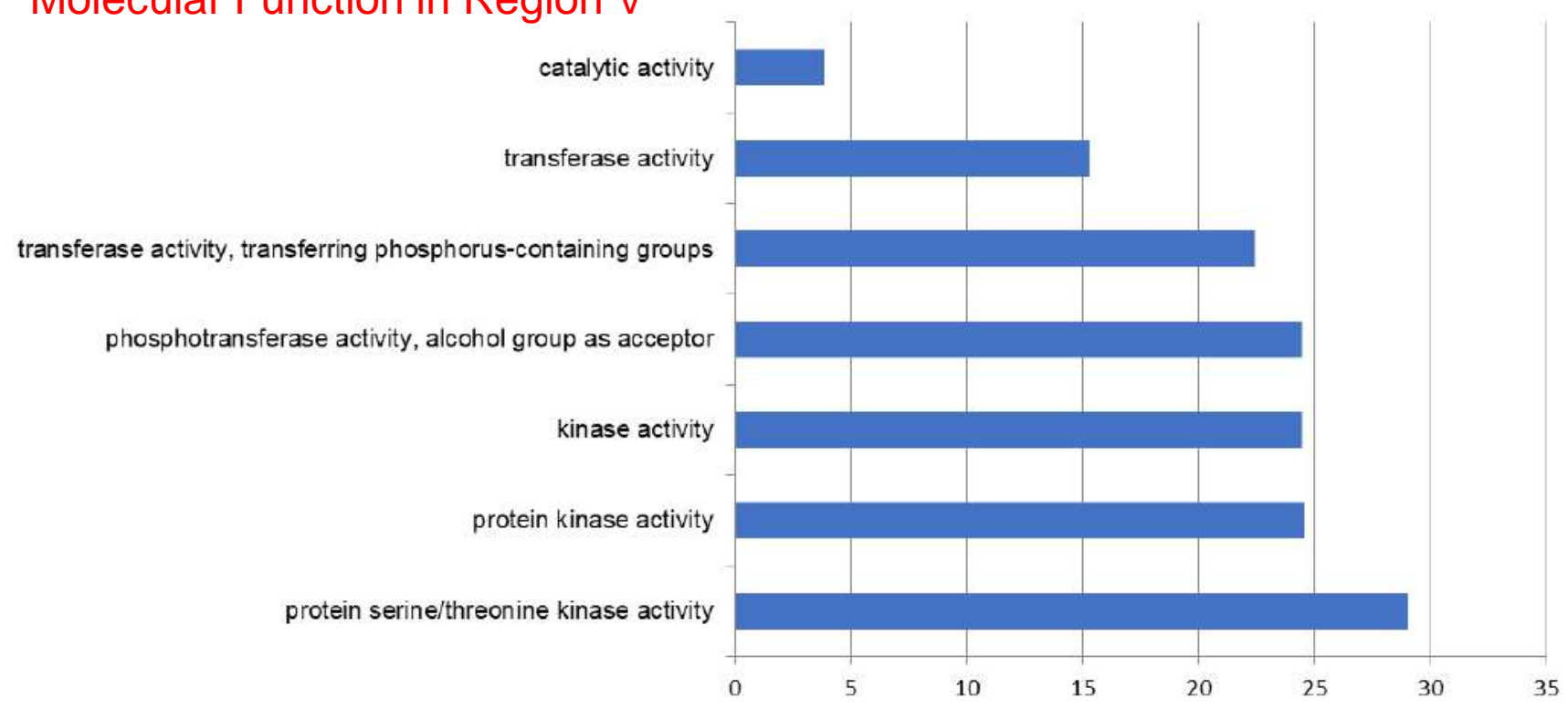




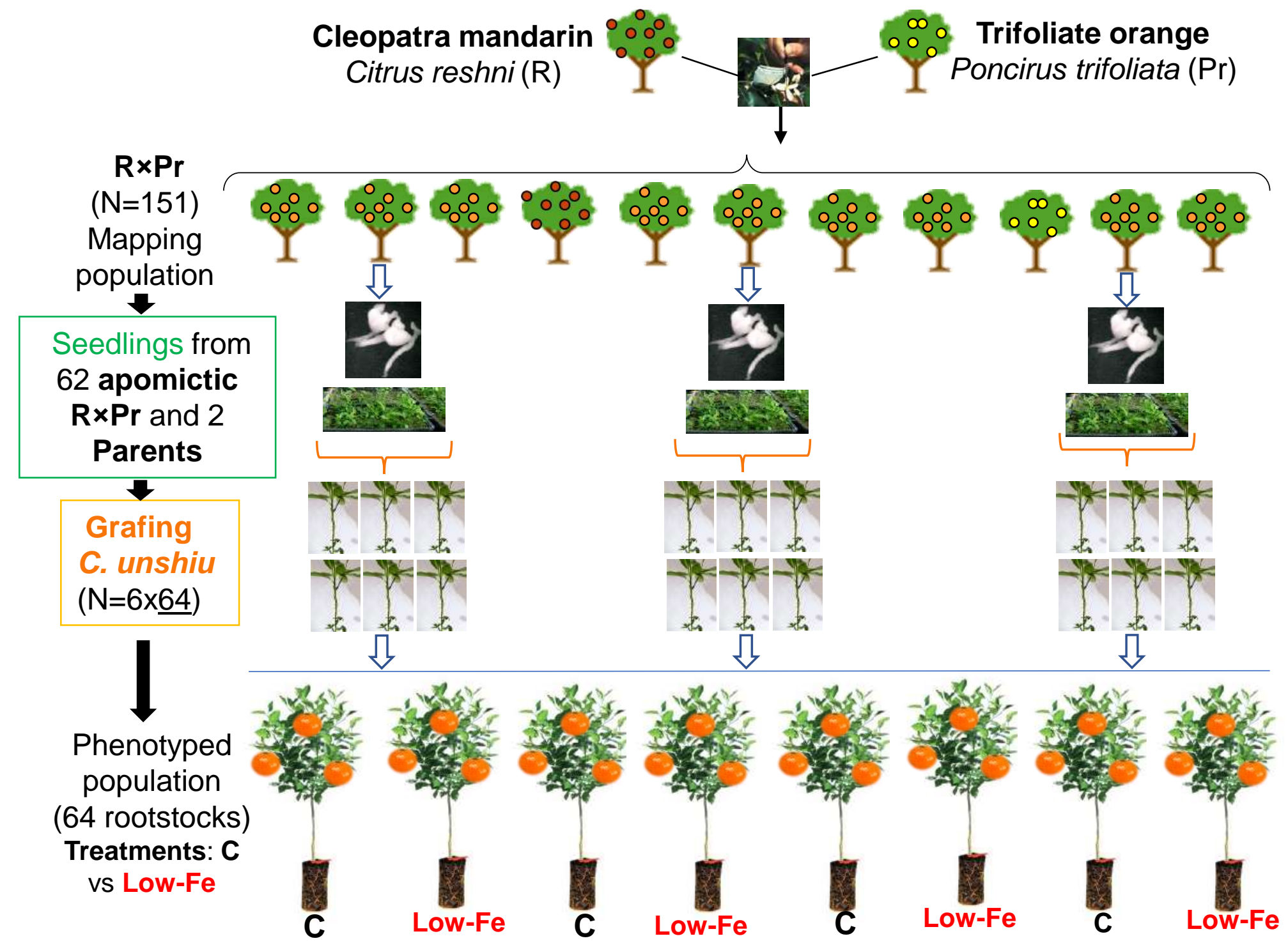



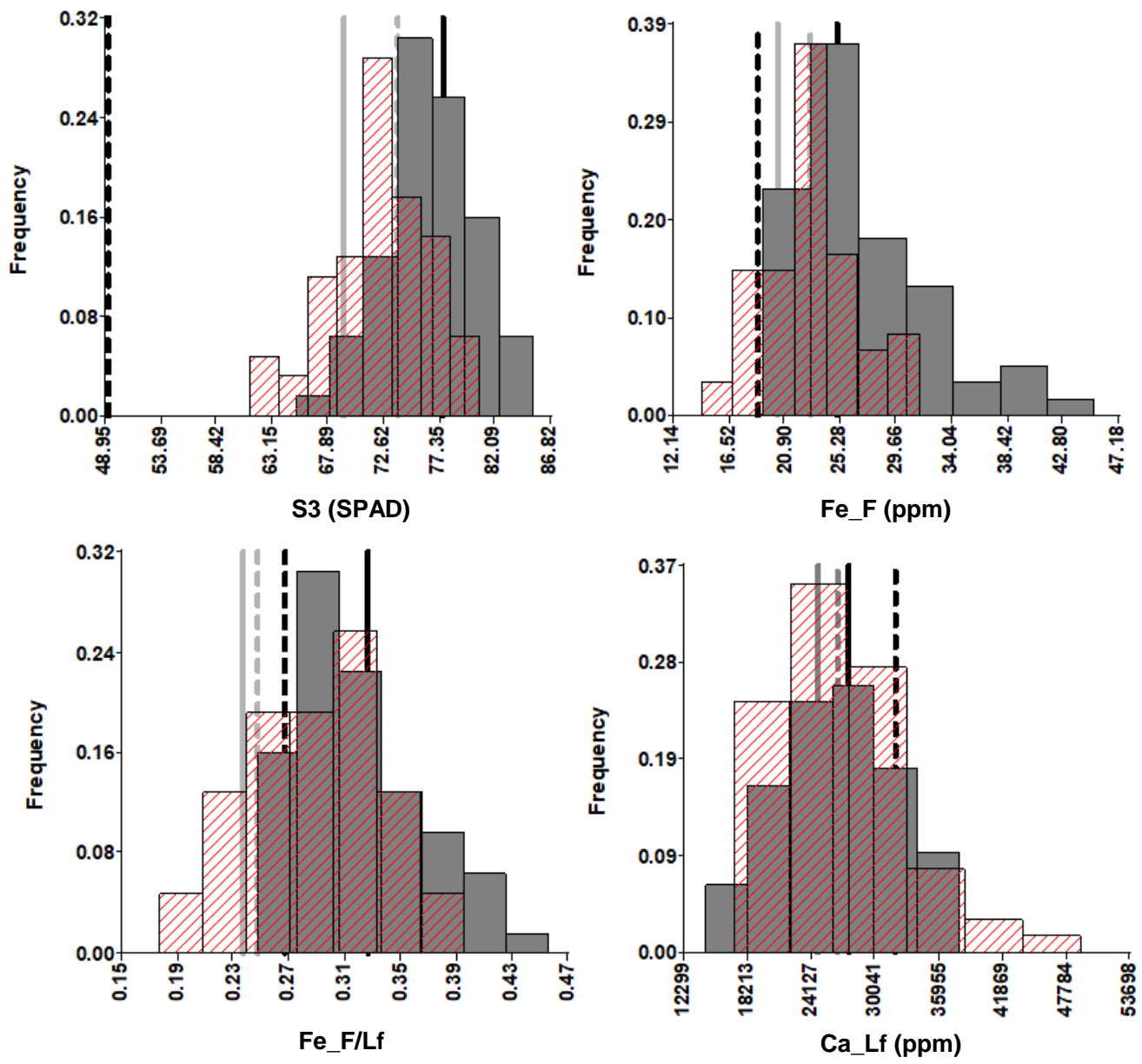


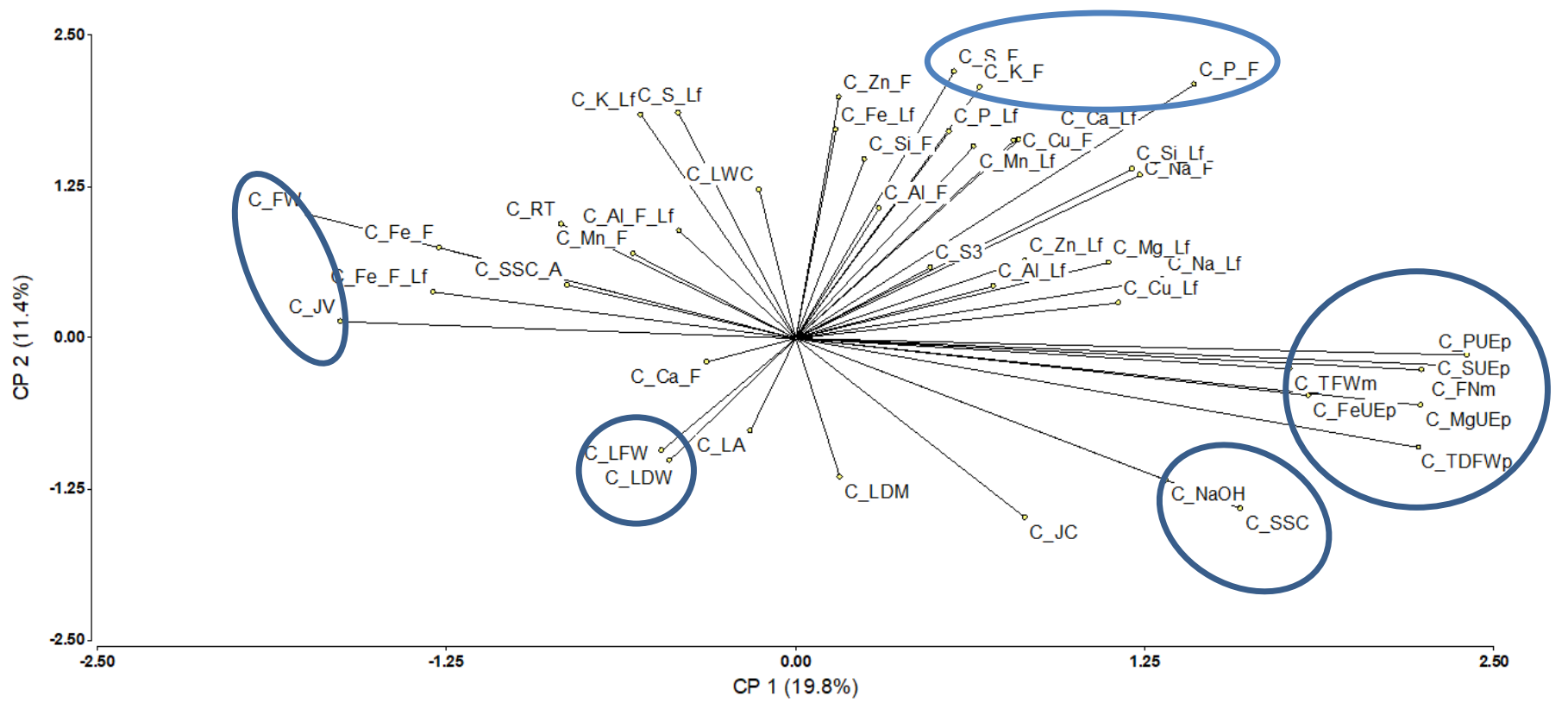




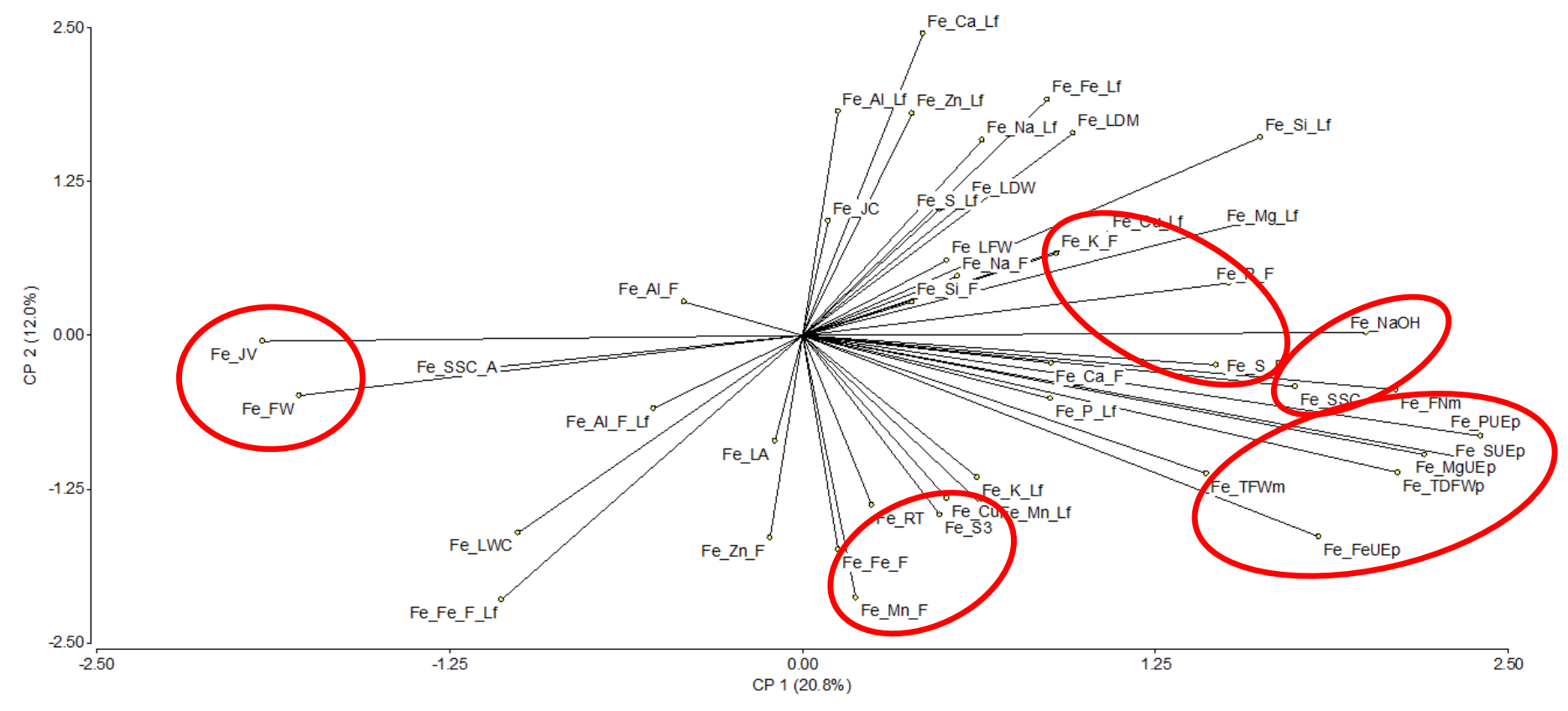




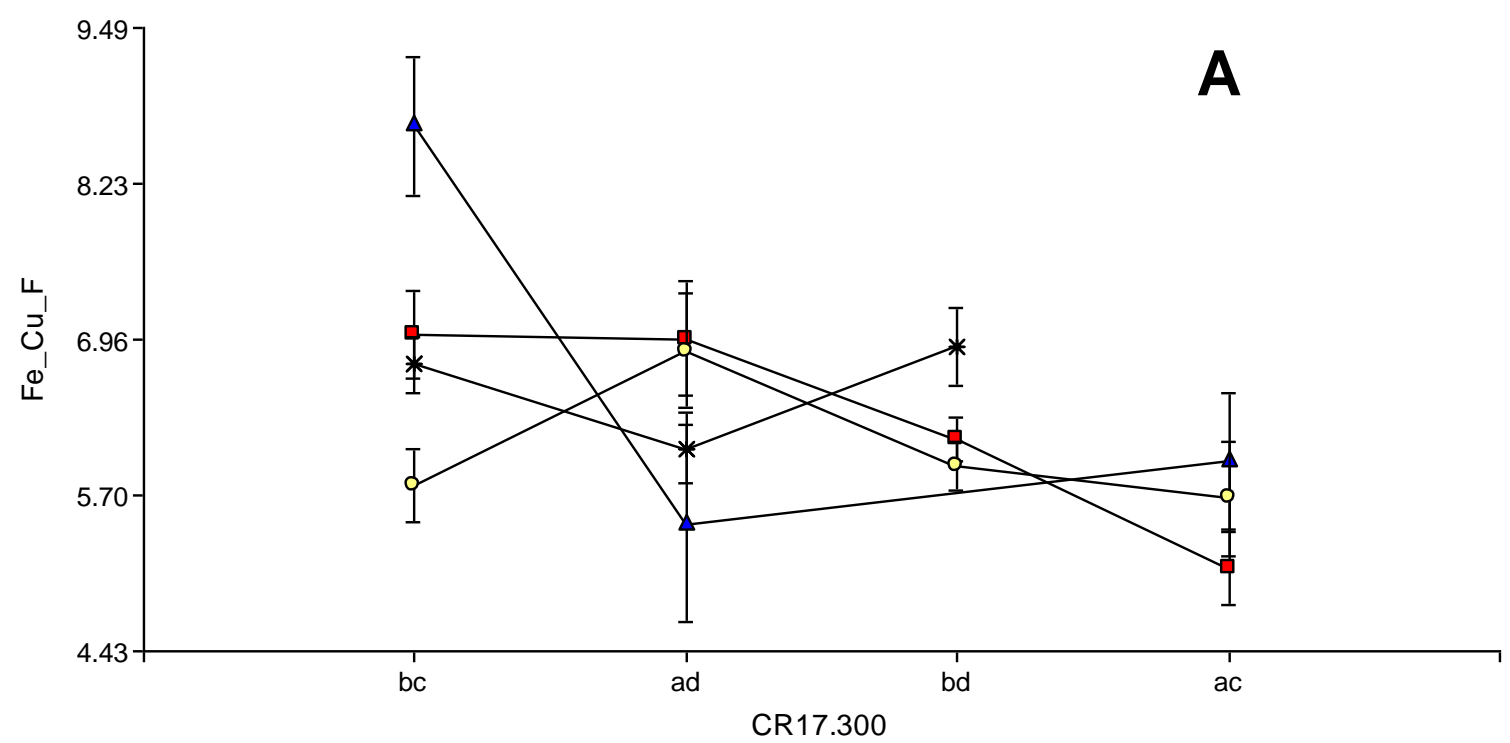

$\longrightarrow$-C8iC1rt.650-ac ד-C8iC1rt.650-ad - C-C8iC1rt.650-bc $\longrightarrow$ C8iC1rt.650-bd

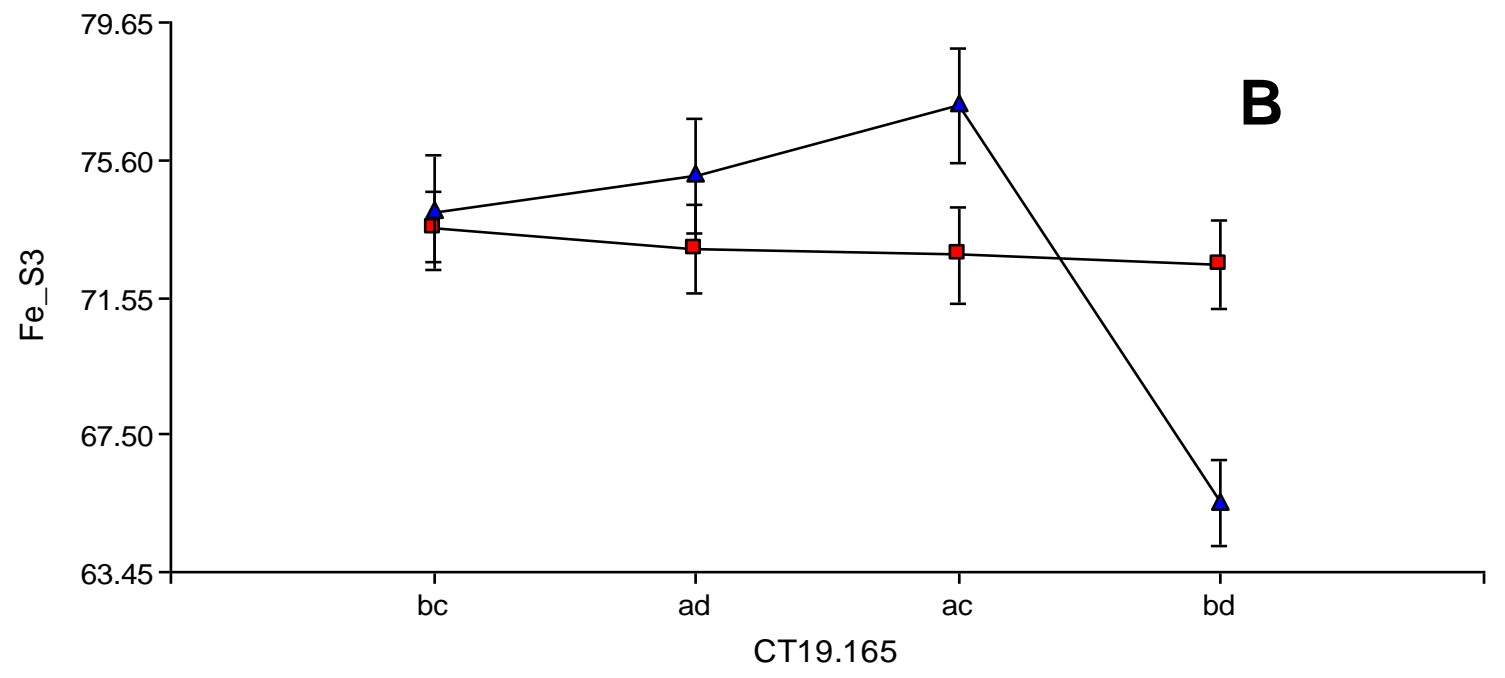

$-\square-C 1,1600-\| \longrightarrow C 1,1600-I m$ 
QTL region II

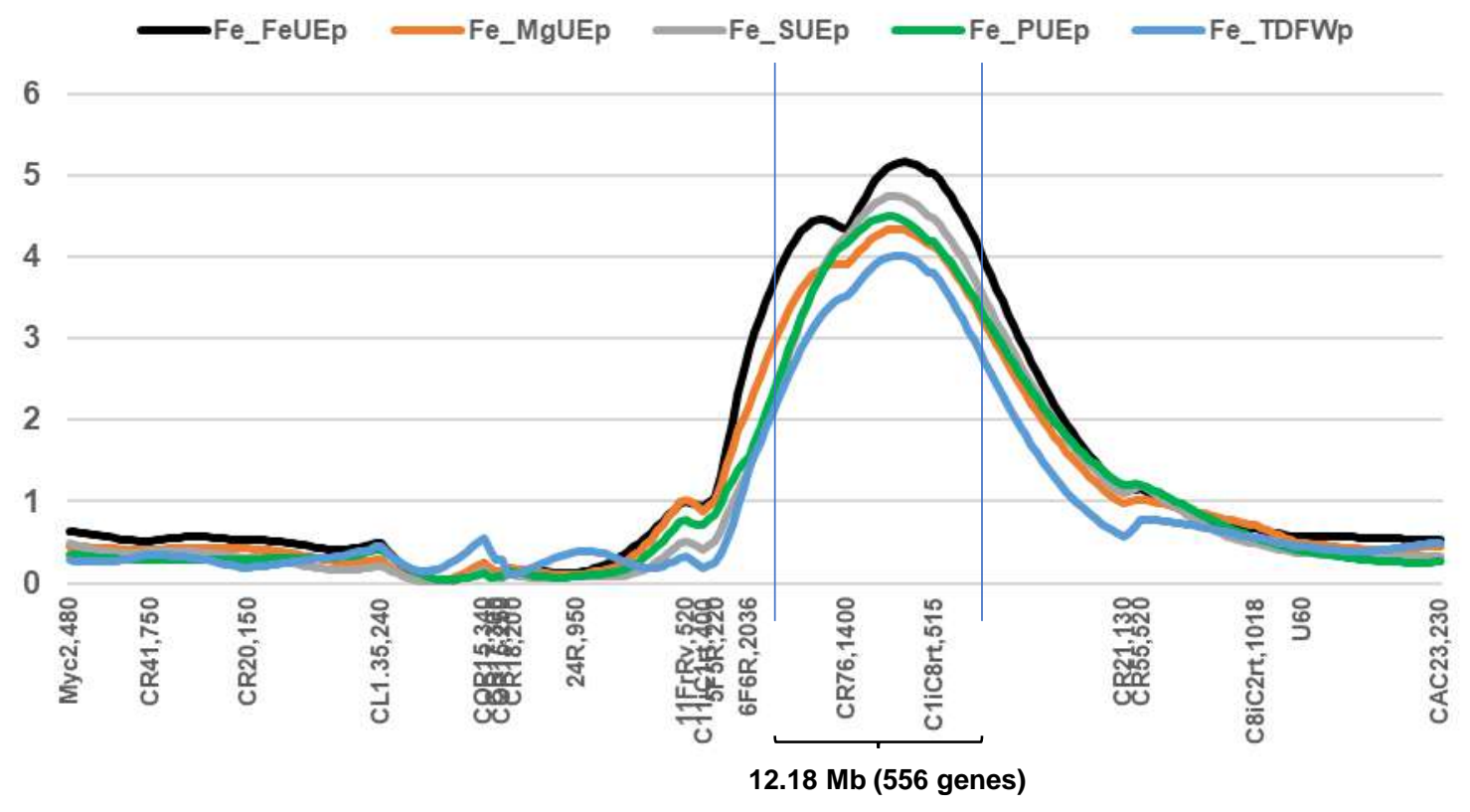

QTL region III

$\longrightarrow \mathrm{Fe}_{-} \mathrm{FNm} \longrightarrow \mathrm{Fe}_{-} \mathrm{TDFWp} \longrightarrow \mathrm{Fe}$-FeUEp $\longrightarrow \mathrm{Fe} \_\mathrm{SUEp} \longrightarrow \mathrm{Fe}_{-} \mathrm{MgUEp} \longrightarrow \mathrm{Fe}$ _PUEp

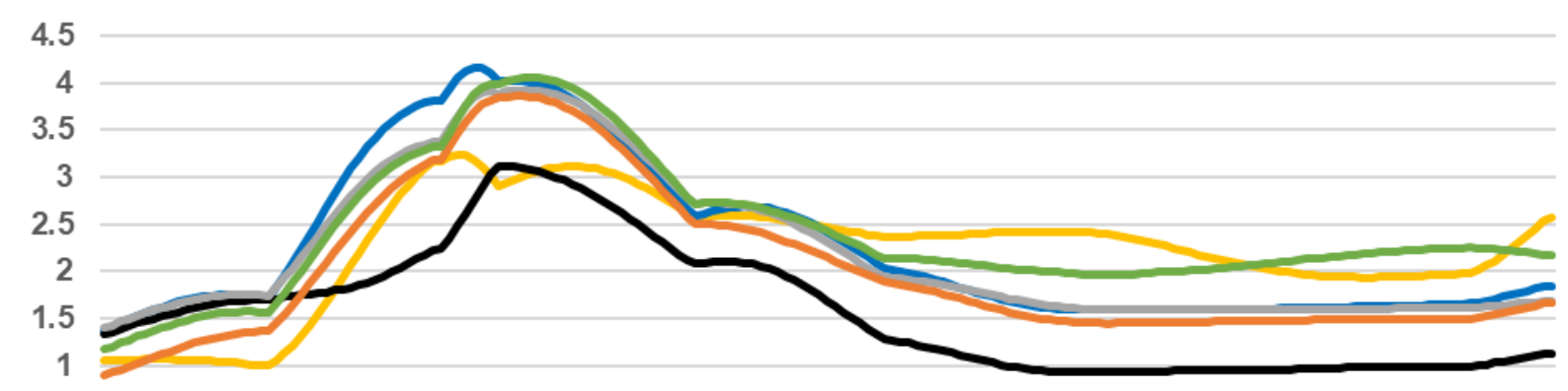

0.5
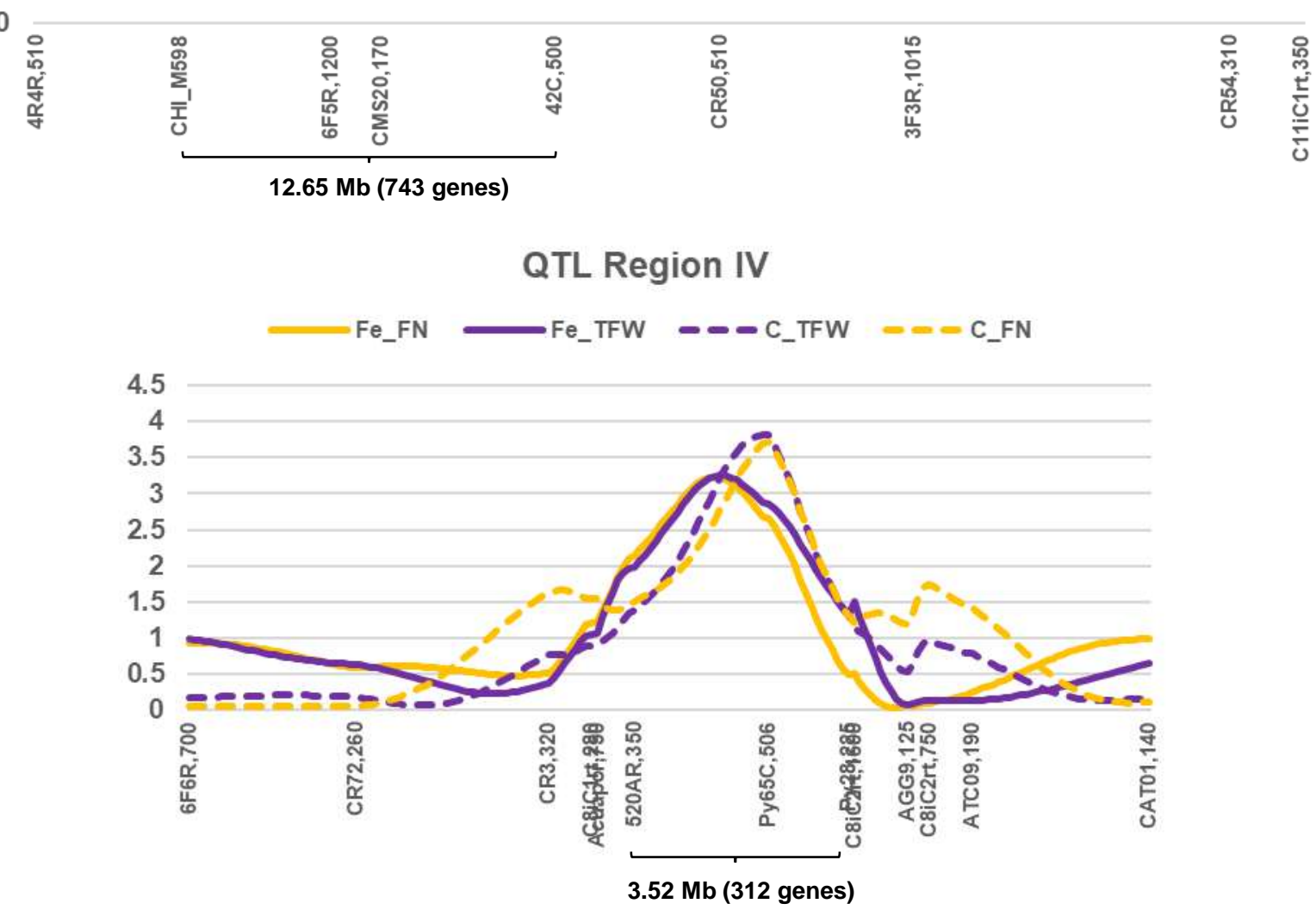